\title{
Variational Inequality Problems with a Continuum of Solutions: Existence and Computation
}

Citation for published version (APA):

Herings, P. J. J., Talman, A. J. J., \& Yang, Z. (2001). Variational Inequality Problems with a Continuum of Solutions: Existence and Computation. Siam Journal on Control and Optimization, 39(6), 1852-1897. https://doi.org/10.1137/S0363012999360592

Document status and date:

Published: 01/01/2001

DOI:

10.1137/S0363012999360592

Document Version:

Publisher's PDF, also known as Version of record

Document license:

Taverne

Please check the document version of this publication:

- A submitted manuscript is the version of the article upon submission and before peer-review. There can be important differences between the submitted version and the official published version of record.

People interested in the research are advised to contact the author for the final version of the publication, or visit the DOI to the publisher's website.

- The final author version and the galley proof are versions of the publication after peer review.

- The final published version features the final layout of the paper including the volume, issue and page numbers.

Link to publication

\footnotetext{
General rights rights.

- You may freely distribute the URL identifying the publication in the public portal. please follow below link for the End User Agreement:

www.umlib.nl/taverne-license

Take down policy

If you believe that this document breaches copyright please contact us at:

repository@maastrichtuniversity.nl

providing details and we will investigate your claim.
}

Copyright and moral rights for the publications made accessible in the public portal are retained by the authors and/or other copyright owners and it is a condition of accessing publications that users recognise and abide by the legal requirements associated with these

- Users may download and print one copy of any publication from the public portal for the purpose of private study or research.

- You may not further distribute the material or use it for any profit-making activity or commercial gain

If the publication is distributed under the terms of Article $25 \mathrm{fa}$ of the Dutch Copyright Act, indicated by the "Taverne" license above, 


\title{
VARIATIONAL INEQUALITY PROBLEMS WITH A CONTINUUM OF SOLUTIONS: EXISTENCE AND COMPUTATION*
}

\author{
P. JEAN-JACQUES HERINGS ${ }^{\dagger}$, DOLF TALMAN TAND $^{\ddagger}$ ZAIFU YANG TA $^{\S}$
}

\begin{abstract}
In this paper three sufficient conditions are provided under each of which an upper semicontinuous point-to-set mapping defined on an arbitrary polytope has a connected set of zero points that connect two distinct faces of the polytope. Furthermore, we obtain an existence theorem of a connected set of solutions to a nonlinear variational inequality problem over arbitrary polytopes. These results follow in a constructive way by designing a new simplicial algorithm. The algorithm operates on a triangulation of the polytope and generates a piecewise linear path of points connecting two distinct faces of the polytope. Each point on the path is an approximate zero point. As the mesh size of the triangulation goes to zero, the path converges to a connected set of zero points linking the two distinct faces. As a consequence, our results generalize Browder's fixed point theorem [Summa Brasiliensis Mathematicae, 4 (1960), pp. 183-191] and an earlier result by the authors [Math. Oper. Res., 21 (1996), pp. 675-696] on the $n$-dimensional unit cube. An application in economics and some numerical examples are also discussed.
\end{abstract}

Key words. polytope, simplicial algorithm, continuum of zero points, system of nonlinear equations, variational inequality, economic equilibrium model

AMS subject classifications. Primary, 49D35, 90A14; Secondary, 90C30, 90C33

PII. S0363012999360592

1. Introduction. Whenever a mathematical model of some phenomenon is constructed, for instance, in engineering or in economics, the first question to ask is whether a solution to the model exists. A very powerful tool that is used to this end is Brouwer's fixed point theorem; see Brouwer [3]. When the model is not a system of equations but a system of correspondences, Kakutani's fixed point theorem [16] is invoked. An alternative to fixed point theorems consists of using intersection theorems on polytopes, with the KKM theorem of Knaster, Kuratowski, and Mazurkiewicz [17] perhaps the most prominent example. It is well known that there is a close relationship between fixed point theorems and intersection theorems. Yet another alternative consists of results that claim the existence of solutions to variational inequality problems, the existence of stationary points, or the existence of zero points.

For certain models, it is not only important to know that there exists at least one solution, but one would like to show the existence of a continuum of solutions. In economics the existence of a continuum of solutions leads to difficulties in expectation formation of agents, and as a consequence provides scope for endogenously generated fluctuations. A particular example comes from general equilibrium theory with price rigidities, where a continuum of solutions on the unit cube as a polytope is shown to

* Received by the editors August 17, 1999; accepted for publication (in revised form) October 18, 2000; published electronically April 18, 2001. This research is part of the Research Program "Competition and Cooperation" of Tilburg University, The Netherlands.

http://www.siam.org/journals/sicon/39-6/36059.html

${ }^{\dagger}$ Department of Economics, University of Maastricht, P.O. Box 616, 6200 MD Maastricht, The Netherlands (P.Herings@algec.unimaas.nl; www.2.unimaas.nl/fdewbae/herings.htm). The research of this author was supported by a fellowship of the Royal Netherlands Academy of Arts and Sciences.

${ }^{\ddagger}$ Department of Econometrics and CentER, Tilburg University, P.O. Box 90153, 5000 LE Tilburg, The Netherlands (Talman@kub.nl).

$\S$ Faculty of Business Administration, Yokohama National University, Yokohama 240-8501, Japan (zyang@business.ynu.ac.jp). The research of this author was supported by Grants-in-Aid of the Ministry of Education, Science, Sports and Culture of Japan, and by a grant of The Netherlands Organization for Scientific Research (NWO). 
exist in Herings [13]. It is therefore important to have generally applicable tools that guarantee the existence of a continuum of zero points to a certain system of equations.

This leads us to the following problem: Given a point-to-set mapping $\varphi: P \Longrightarrow$ $\mathbb{R}^{n}$, with $P$ an arbitrary polytope, what reasonable conditions can guarantee the existence of a continuum of solutions $x$ to the system

$$
0^{n} \in \varphi(x) \text {, }
$$

where $0^{n}$ denotes the $n$-dimensional vector of zeros? Our approach to show the existence of a continuum of solutions is to show that there is a connected subset of solutions that links together at least two distinct points, thereby guaranteeing the continuum. In this paper we will show that any upper semicontinuous point-to-set mapping with some mild (boundary) conditions will have a connected set of zero points linking together two distinct faces of the polytope $P$.

It is well known that under certain conditions a point-to-set mapping defined on a polytope has a solution to the variational inequality problem. We generalize the variational inequality problem and define a parametric variational inequality problem. In this paper we show that under similar conditions a point-to-set mapping defined on a polytope $P$ has a connected set of solutions to the parametric variational inequality problem, called parametrized stationary points. The set of parametrized stationary points connects two distinct faces of $P$. With respect to some given nonzero vector $c$, on one of these faces, denoted by $F^{-}$, the value $c^{\top} x$ is minimized for $x \in P$, while on the other face, denoted by $F^{+}$, the value $c^{\top} x$ is maximized for $x \in P$. A special case occurs when both $F^{-}$and $F^{+}$are vertices of $P$ and the set of parametrized stationary points contains both these vertices. Under the three different conditions the set of parametrized stationary points is a connected set of zero points linking the two distinct faces $F^{-}$and $F^{+}$of $P$.

We prove the existence results by designing a simplicial variable dimension algorithm on a polytope. This type of algorithm was initiated by Scarf [22]. Simplicial homotopy methods were developed by Eaves [8]. The simplicial restart variable dimension algorithm was introduced by van der Laan and Talman [18] to compute a fixed point of a continuous function from the unit simplex into itself. Such an algorithm generates a unique sequence of simplices of varying dimension in a simplicial subdivision of the set and connects the arbitrarily chosen starting point with an approximate solution. For other recent developments, we refer to Talman and Yamamoto [24], Yamamoto [26], Brown, DeMarzo, and Eaves [5], DeMarzo and Eaves [6], Yang $[27,28]$, and van der Laan, Talman, and Yang [19]. Allgower and Georg [1], Todd [25], and Yang [28] provide comprehensive treatments of simplicial algorithms.

In this paper a simplicial algorithm is proposed which generates within a simplicial subdivision of $P$ a finite sequence of simplices of varying dimension. This sequence connects two different simplices, one simplex lying in the face $F^{-}$of $P$ and the other lying in the face $F^{+}$of $P$. The sequence of simplices connecting these two simplices is generated by the algorithm through a sequence of semilexicographic pivot steps in a linear system of equations. In case the face $F^{-}$is not a vertex of $P$, the algorithm starts by finding a suitable simplex in $F^{-}$. Next it generates a sequence of simplices of varying dimension in $P$. It is possible that the algorithm returns to $F^{-}$. Then it generates a third simplex in $F^{-}$from where a sequence of adjacent simplices in $P$ is generated. It is shown that the algorithm eventually finds a simplex in $F^{-}$from which a sequence of adjacent simplices reaching $F^{+}$is generated.

Induced by the sequence of adjacent simplices, the algorithm yields a piecewise linear path of parametrized stationary points of a piecewise linear approximation of 
the underlying mapping. When the mesh size of the simplicial subdivision of $P$ goes to zero the sequence (or at least a subsequence) of piecewise linear paths converges to a connected set of parametrized stationary points of the original mapping. This set has points in common with both faces $F^{-}$and $F^{+}$.

The results in the paper generalize earlier results of Browder [4], Mas-Colell [20], and Herings, Talman, and Yang [15]. In the case of Browder's theorem the polytope is the Cartesian product of a polytope of one dimension less and the unit interval $[0,1]$, while $c$ is the unit vector with the one on the last position. Mas-Colell's result is an extension of Browder's result to deal with correspondences. Both Browder and MasColell proved their results via a rather sophisticated machinery. Since our approach here is constructive, we therefore also obtain an alternative but constructive proof for their results. In Herings, Talman, and Yang [15] the polytope equals the unit cube and the vector $c$ is the vector of ones. In both Browder's and Mas-Colell's theorems, a connected set of fixed points is obtained connecting the levels 0 and 1 , whereas the result on the unit cube yields a connected set of zero points connecting the vector of zeros and the vector of ones.

Intersection theorems with a continuum of intersection points can be found in Freidenfelds [9] and Herings and Talman [14]. Although Freidenfelds's intersection theorem typically has a continuum of intersection points, this is not necessarily the case. Freidenfelds's result generalizes the intersection theorem of Scarf [22]. Herings and Talman's results generalize a number of intersection theorems on the unit simplex, including the Scarf-result and the KKM-result, to intersection theorems on the unit cube and show the existence of a continuum of intersection points. The reader should be aware that compared with a large amount of existence results for a single fixed or zero point, existence results for a continuum of fixed or zero points are very rare.

This paper is organized as follows. In section 2 we state the problem and in section 3 we give three sufficient conditions for the existence of a connected set of zero points of an upper semicontinuous point-to-set mapping over an arbitrary polytope which link together two distinct faces of the polytope. An example is also given. In section 4 we introduce the algorithm, prove its convergence, and illustrate the algorithm by an example. In section 5 we analyze the accuracy of the approximation of zero points and prove the existence theorems. In section 6 we discuss a more general case. In section 7 we derive as special cases Browder's and Mas-Colell's theorems and an earlier result of the authors on the unit cube, and we also give an interesting economic application.

2. The problem. Let $I_{m}$ denote the set of the first $m$ positive integers. Consider an arbitrary full-dimensional polytope $P$ that has the following representation as a polyhedron:

$$
P=\left\{x \in \mathbb{R}^{n} \mid a^{i \top} x \leq b_{i} \forall i \in I_{m}\right\} .
$$

For each subset $I$ of $I_{m}$, define

$$
F(I)=\left\{x \in P \mid a^{i \top} x=b_{i} \forall i \in I\right\} .
$$

Then $F(I)$ is called a face of $P$ unless it is empty. Note that $F(\emptyset)=P$. Let

$$
\mathcal{I}=\left\{I \subset I_{m} \mid F(I) \text { is a face of } P\right\} .
$$

A face $F$ of the set $F(I)$ of dimension one lower than the dimension of $F(I)$ is called a facet of $F(I)$. The polytope $P$ is said to be simple if the dimension of any face $F(I)$ of $P$ is equal to $n-|I|$. Throughout the paper, whenever we use a polytope 
$P$, it is assumed that $P$ is simple and that its representation as a polyhedron has no redundant constraints.

We have the following observations.

(i) For each face $F$ of $P$, the set $I \in \mathcal{I}$ with $F=F(I)$ is unique and is given by the set $\left\{i \in I_{m} \mid a^{i \top} x=b_{i} \forall x \in F\right\}$.

(ii) The set $F(I)$ is a vertex of $P$ if and only if $I \in \mathcal{I}$ with $|I|=n$.

(iii) If $I \in \mathcal{I}$, then $I \backslash\{i\} \in \mathcal{I}$ for any $i \in I$.

(iv) For some $I \in \mathcal{I}, F$ is a facet of $F(I)$ if and only if $F=F(I \cup\{i\})$ for some $i \notin I$ with $I \cup\{i\} \in \mathcal{I}$.

(v) For any $I \in \mathcal{I}$, the vectors $a^{i}$ with $i \in I$ are linearly independent.

Let $c$ be an arbitrary nonzero vector in $\mathbb{R}^{n}$. Then $F^{+}$will denote the face of $P$ such that for each $x^{+} \in F^{+}$it holds that $c^{\top} x^{+}=\max _{x \in P} c^{\top} x$, and $F^{-}$will denote the face of $P$ such that for each $x^{-} \in F^{-}$it holds that $c^{\top} x^{-}=\min _{x \in P} c^{\top} x$. Let $t^{+}=c^{\top} x^{+}$for $x^{+} \in F^{+}$and $t^{-}=c^{\top} x^{-}$for $x^{-} \in F^{-}$. Since $P$ is full-dimensional, it follows that $t^{-}<t^{+}$and therefore $F^{-} \cap F^{+}=\emptyset$. We define

$$
\begin{aligned}
& I^{+}=\left\{i \in I_{m} \mid a^{i \top} x=b_{i} \forall x \in F^{+}\right\}, \\
& I^{-}=\left\{i \in I_{m} \mid a^{i \top} x=b_{i} \forall x \in F^{-}\right\} .
\end{aligned}
$$

So $F^{+}=F\left(I^{+}\right)$and $F^{-}=F\left(I^{-}\right)$.

We need some further notation. For each $I \in \mathcal{I}$, we define

$$
\begin{aligned}
A(I) & =\left\{y \in \mathbb{R}^{n} \mid y=\sum_{i \in I} \mu_{i} a^{i}+\beta c, \mu_{i} \geq 0 \forall i \in I, \text { and } \beta \in \mathbb{R}\right\}, \\
A_{0}(I) & =\left\{y \in \mathbb{R}^{n} \mid y=\sum_{i \in I} \mu_{i} a^{i}, \mu_{i} \geq 0 \forall i \in I\right\}, \\
A^{*}(I) & =\left\{x \in \mathbb{R}^{n} \mid x^{\top} y \leq 0 \forall y \in A(I)\right\}, \\
A_{0}^{*}(I) & =\left\{x \in \mathbb{R}^{n} \mid x^{\top} y \leq 0 \forall y \in A_{0}(I)\right\} .
\end{aligned}
$$

Note that $A(\emptyset)=\left\{y \in \mathbb{R}^{n} \mid y=\beta c, \beta \in \mathbb{R}\right\}, A_{0}(\emptyset)=\left\{0^{n}\right\}, A^{*}(\emptyset)=\left\{x \in \mathbb{R}^{n} \mid\right.$ $\left.x^{\top} c=0\right\}$, and $A_{0}^{*}(\emptyset)=\mathbb{R}^{n}$. Moreover, for any $I \in \mathcal{I}$ we have that $A_{0}(I) \subset A(I)$, $A^{*}(I) \subset A_{0}^{*}(I), A^{*}(I) \cap A(I)=\left\{0^{n}\right\}$, and $A_{0}^{*}(I) \cap A_{0}(I)=\left\{0^{n}\right\}$. We may interpret $A(I)$ and $A_{0}(I)$ as normal cones to the boundary of $P$ and $A^{*}(I)$ and $A_{0}^{*}(I)$ as tangent cones. The pairs $\left(A(I), A^{*}(I)\right)$ and $\left(A_{0}(I), A_{0}^{*}(I)\right)$ may be viewed as primal and dual pairs as well; see, e.g., Aubin [2].

Let $\varphi: P \Longrightarrow \mathbb{R}^{n}$ be a correspondence that satisfies the following assumption.

Assumption 2.1. The correspondence $\varphi: P \Longrightarrow \mathbb{R}^{n}$ is nonempty valued, compact valued, convex valued, and upper semicontinuous.

For an arbitrary function $f: P \rightarrow \mathbb{R}^{n}$, the stationary point (or variational inequality) problem for $f$ on the polytope $P$ is to find a point $x^{*} \in P$ such that

$$
\left(x^{*}-x\right)^{\top} f\left(x^{*}\right) \geq 0 \forall x \in P .
$$

Such a point $x^{*}$ is called a stationary point of $f$ on $P$. It is well known that a continuous function on a convex compact set has a stationary point; see Hartman and Stampacchia [11] and Eaves [8].

In the following we give the definition of a stationary point of a correspondence on the polytope $P$ with respect to the vector $c$. 
Definition 2.2. A point $x^{*} \in P$ is a stationary point of a correspondence $\varphi$ on the polytope $P$ with respect to the nonzero vector $c$ if there exists $f \in \varphi\left(x^{*}\right)$ such that $\left(x^{*}-x\right)^{\top} f \geq 0 \forall x \in P$ satisfying $c^{\top} x=c^{\top} x^{*}$.

We call the problem of finding a stationary point with respect to a nonzero vector $c$ a parametric variational inequality problem. A solution to it is called a parametrized stationary point. Now we have the following simple but important observation.

Lemma 2.3. A point $x^{*} \in P$ is a parametrized stationary point of the mapping $\varphi$ on $P$ with respect to $c$ if and only if for some $I \in \mathcal{I}$ it holds that $x^{*} \in F(I)$ and $\varphi\left(x^{*}\right) \cap A(I) \neq \emptyset$.

Proof. Let $x^{*}$ be a parametrized stationary point of $\varphi$ on $P$ with respect to $c$. Hence there exists $f \in \varphi\left(x^{*}\right)$ such that $\left(x^{*}-x\right)^{\top} f \geq 0 \forall x \in P$ satisfying $c^{\top} x=c^{\top} x^{*}$; i.e., $x^{*}$ maximizes $x^{\top} f$ subject to $a^{i \top} x \leq b_{i}, i \in I_{m}$, and $c^{\top} x=c^{\top} x^{*}$. Therefore, there exist $\mu_{i} \geq 0, i \in I_{m}$, and $\beta \in \mathbb{R}$ satisfying

$$
f=\sum_{i \in I_{m}} \mu_{i} a^{i}+\beta c
$$

and $\mu_{i}=0$ if $a^{i \top} x^{*}<b_{i}$. Let $I=\left\{i \in I_{m} \mid a^{i \top} x^{*}=b_{i}\right\}$. Then $x^{*} \in F(I)$ and $f \in A(I) \cap \varphi\left(x^{*}\right)$.

Next let $x^{*} \in P$ be such that $x^{*} \in F(I)$ and $A(I) \cap \varphi\left(x^{*}\right) \neq \emptyset$ for some $I \in \mathcal{I}$. Take any $f \in A(I) \cap \varphi\left(x^{*}\right)$. Then $f \in \varphi\left(x^{*}\right)$ and there exists $\mu_{i} \geq 0, i \in I$, and $\beta \in \mathbb{R}$ satisfying

$$
f=\sum_{i \in I} \mu_{i} a^{i}+\beta c .
$$

Note that $a^{i \top} x^{*}=b_{i} \forall i \in I$, and for any $x \in P, a^{i \top} x \leq b_{i} \forall i \in I$. Thus $x^{* \top} f=$ $\sum_{i \in I} \mu_{i} b_{i}+\beta c^{\top} x^{*} \geq x^{\top} f$ for all $x \in P$ with $x^{\top} c=x^{* \top} c$. By definition, $x^{*}$ is a stationary point of $\varphi$ on $P$ with respect to $c$.

We show the existence of a continuum of parametrized stationary points of the correspondence $\varphi$ with respect to the vector $c$. A point $x^{*} \in P$ is called a zero point of the mapping $\varphi$ if $0^{n} \in \varphi\left(x^{*}\right)$. Any zero point of $\varphi$ is a parametrized stationary point. The main purpose of this paper is to find conditions on $\varphi$ which enable us to guarantee the existence and the computation of a continuum of zero points of a correspondence $\varphi$ on $P$ such that it contains points of both $F^{-}$and $F^{+}$.

3. Existence conditions. The first result is on the existence of parametrized stationary points.

TheOREM 3.1. Let $\varphi: P \Longrightarrow \mathbb{R}^{n}$ be any correspondence satisfying Assumption 2.1 and let $c \in \mathbb{R}^{n} \backslash\left\{0^{n}\right\}$ be given. Then there exists a connected set $C$ of parametrized stationary points of $\varphi$ on $P$ with respect to $c$ such that $C \cap F^{-} \neq \emptyset$ and $C \cap F^{+} \neq \emptyset$.

The theorem makes clear that there are many parametrized stationary points. To be more precise, there exists a continuum of them with a special topological structure. The set of parametrized stationary points has a connected subset that links the two faces $F^{-}$and $F^{+}$.

In order to obtain the existence of a continuum of zero points, we need to impose certain conditions on the correspondence $\varphi$. The following three results list sufficient conditions for the existence of a continuum of zero points of $\varphi$.

Theorem 3.2. Let $\varphi: P \Longrightarrow \mathbb{R}^{n}$ be any correspondence satisfying Assumption 2.1 and let $c \in \mathbb{R}^{n} \backslash\left\{0^{n}\right\}$. If for any $x \in F(I), I \in \mathcal{I}$, it holds that $\varphi(x) \cap A(I) \subset$ $\left\{0^{n}\right\}$, then there exists a connected set $C$ of zero points of $\varphi$ such that $C \cap F^{-} \neq \emptyset$ and $C \cap F^{+} \neq \emptyset$. 
The condition in the theorem states that for any $x$ in the face $F(I)$ of $P$ the image $\varphi(x)$ may not have nonzero elements in common with $A(I)$.

Theorem 3.3. Let $\varphi: P \Longrightarrow \mathbb{R}^{n}$ be any correspondence satisfying Assumption 2.1 and let $c \in \mathbb{R}^{n} \backslash\left\{0^{n}\right\}$. If for any $x \in F(I), I \in \mathcal{I}$, it holds that

(i) $\varphi(x) \cap A_{0}^{*}(I) \cap A(I) \subset\left\{0^{n}\right\}$,

(ii) $\varphi(x) \cap A_{0}^{*}(I) \neq \emptyset$,

then there exists a connected set $C$ of zero points of $\varphi$ such that $C \cap F^{-} \neq \emptyset$ and $C \cap F^{+} \neq \emptyset$.

The conditions in the theorem imply that for any $x$ in the face $F(I)$ of $P$ the image $\varphi(x)$ may not have nonzero elements in common with $A(I) \cap A_{0}^{*}(I)$ and that at least one element of $\varphi(x)$ lies in $A_{0}^{*}(I)$.

TheOrem 3.4. Let $\varphi: P \Longrightarrow \mathbb{R}^{n}$ be any correspondence satisfying Assumption 2.1, and let $c \in \mathbb{R}^{n} \backslash\left\{0^{n}\right\}$. If for any $x \in F(I), I \in \mathcal{I}$, it holds that

$$
\varphi(x) \cap A^{*}(I) \neq \emptyset \text {, }
$$

then there exists a connected set $C$ of zero points of $\varphi$ such that $C \cap F^{-} \neq \emptyset$ and $C \cap F^{+} \neq \emptyset$.

The condition in the theorem says that for any $x$ in the face $F(I)$ of $P$ at least one element of $\varphi(x)$ lies in $A^{*}(I)$.

The three theorems state different conditions for which a continuum of zero points can be shown to exist. Moreover, there is a logical order in these sufficient conditions. Theorem 3.2 states a weak condition, but one that holds for all elements of $\varphi(x)$. Theorem 3.4 gives a strong condition but requires this condition to hold for only one element in $\varphi(x)$. Theorem 3.3 is in between: it gives a very weak condition for all elements in $\varphi(x)$ together with a rather weak condition for some element in $\varphi(x)$.

The following result claims that for the special case where $\varphi$ is a function $f$, Theorem 3.2 is the strongest and Theorem 3.4 the weakest. Note that Assumption 2.1 implies that the function $f$ is continuous.

Theorem 3.5. Let $P$ be any polytope and let $c \in \mathbb{R}^{n} \backslash\left\{0^{n}\right\}$. The collection of functions satisfying the conditions of Theorem 3.2 contains the collection of functions satisfying the conditions of Theorem 3.3, which contains the collection of functions satisfying the conditions of Theorem 3.4 .

Proof. Suppose that a function $f$ from $P$ to $\mathbb{R}^{n}$ satisfies the conditions of Theorem 3.4. Take any $x$ in $F(I)$, so $f(x) \in A^{*}(I)$. Since $A^{*}(I) \subset A_{0}^{*}(I)$ and $A(I) \cap A^{*}(I)=\left\{0^{n}\right\}$ we obtain that $f(x) \in A_{0}^{*}(I)$ and $f(x)$ not in $A(I)$ unless $f(x)=0^{n}$. Hence the conditions of Theorem 3.3 are satisfied. Suppose now that a function satisfies the conditions of Theorem 3.3. Again take any $x$ in $F(I)$, so $f(x) \in A_{0}^{*}(I)$ and $f(x)$ not in $A(I) \cap A_{0}^{*}(I)$ unless $f(x)=0^{n}$. Hence $f(x)$ not in $A(I)$ unless $f(x)=0^{n}$.

That Theorems 3.2, 3.3, and 3.4 are mutually exclusive for correspondences follows from the fact that in case of correspondences the image $\varphi(x)$ of any point $x$ in $P$ might consist of more than one element. For example, for a point $x$ in the face $F(I)$ of $P$ it is required in Theorem 3.2 that no nonzero element of $\varphi(x)$ lies in $A(I)$ which does not imply that at least one such element should lie in $A^{*}(I)$ as required in the conditions of Theorem 3.4. On the other hand if for an $x$ in $F(I)$ it holds that some nonzero element $f \in \varphi(x)$ lies in $A^{*}(I)$, as in the conditions of Theorem 3.4, then this implies that $f$ indeed does not lie in $A(I)$ but not necessarily that all the other elements of $\varphi(x)$ also do not lie in $A(I)$ as required in Theorem 3.2. Similar remarks can be made when comparing the conditions of Theorem 3.3 with the conditions in the other two theorems. 


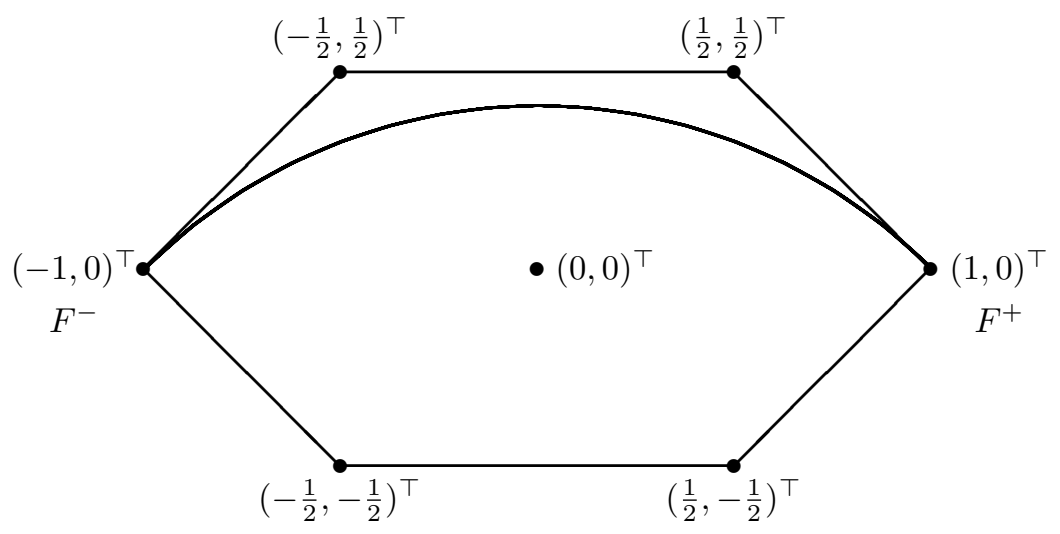

FIG. 3.1. The set of zero points of $f$ on $P$ in Example 3.1 linking $F^{-}$and $F^{+}$.

The theorems above will be proved in a constructive manner in the following sections. Their applications will be discussed in section 7. To conclude this section we will give a numerical example to illustrate the existence of a connected set of zero points on a two-dimensional diamond and then we will illustrate the algorithm with this example in the next section.

Example 3.1. Let the polytope $P$, illustrated in Figure 3.1, be given by

$$
P=\left\{x \in \mathbb{R}^{2}|| x_{2}|\leq 1 / 2,| x_{1}|+| x_{2} \mid \leq 1\right\} .
$$

The vector $c$ and the function $f$ on $P$ are given by $c=(1,0)^{\top}$ and

$$
f(x)=\left(2-x_{1}^{2}-\left(x_{2}+1\right)^{2}, \sqrt{2-x_{1}^{2}}-x_{2}-1\right)^{\top} .
$$

Clearly, $F^{-}$and $F^{+}$correspond to $(-1,0)^{\top},(1,0)^{\top}$, vertices of $P$, respectively. Furthermore, if $x$ lies on the boundary of $P$, then $f_{1}(x)>0$ and $f_{2}(x)>0$ when $x_{2}<0$, and $f_{1}(x)<0$ and $f_{2}(x)<0$ when $x_{2}>0$. Also, $f_{1}(x)=0$ whenever $f_{2}(x)=0$. Note that $P$ can be rewritten as

$$
P=\left\{x \in \mathbb{R}^{2} \quad \mid \begin{array}{l}
\quad x_{1}+x_{2} \leq 1, x_{2} \leq 1 / 2,-x_{1}+x_{2} \leq 1, \\
\\
\left.-x_{1}-x_{2} \leq 1,-x_{2} \leq 1 / 2, x_{1}-x_{2} \leq 1\right\} .
\end{array}\right.
$$

One can easily verify that $f$ is continuous and satisfies the condition of Theorem 3.2. So it follows that there exists a connected set of zero points of $f$ on $P$ linking both $(-1,0)^{\top}$ and $(1,0)^{\top}$. In fact this set equals the circle segment $\left\{x \in P \mid x_{1}^{2}+\left(x_{2}+1\right)^{2}=\right.$ $2\}$; see Figure 3.1 .

4. The algorithm and its convergence proof. In this section we describe an algorithm on the polytope $P$ that generates a piecewise linear path of parametrized stationary points, with respect to the given nonzero vector $c$, of a piecewise linear approximation of the mapping $\varphi$. The piecewise linear approximation is taken with respect to some simplicial subdivision of the set $P$. In this and the following sections we assume that the faces $F^{-}$and $F^{+}$are vertices of $P$, denoted by $x^{-}$and $x^{+}$, respectively. In that case the piecewise linear path generated by the algorithm contains both $x^{-}$and $x^{+}$and can be traced by a sequence of semilexicographic pivot steps in a system of linear equations. The general case is discussed in section 6 . 
For a nonnegative integer $t$, a $t$-dimensional simplex or $t$-simplex, denoted by $\sigma$, is defined by the convex hull of $t+1$ affinely independent points $x^{1}, \ldots, x^{t+1}$ in $\mathbb{R}^{n}$. We often write $\sigma=\sigma\left(x^{1}, \ldots, x^{t+1}\right)$ and call $x^{1}, \ldots, x^{t+1}$ the vertices of $\sigma$. A $(t-1)$-simplex being the convex hull of $t$ vertices of $\sigma$ is said to be a facet of $\sigma$. The facet $\tau\left(x^{1}, \ldots, x^{i-1}, x^{i+1}, \ldots, x^{t+1}\right)$ is called the facet of $\sigma\left(x^{1}, \ldots, x^{t+1}\right)$ opposite to the vertex $x^{i}$. For $k, 0 \leq k \leq t$, a $k$-simplex being the convex hull of $k+1$ vertices of $\sigma$ is said to be a $k$-face or face of $\sigma$. A finite collection $\mathcal{T}$ of $n$-simplices is a triangulation of the polytope $P$ if

(i) $P$ is the union of all simplices in $\mathcal{T}$;

(ii) the intersection of any two simplices of $\mathcal{T}$ is either the empty set or a common face of both.

Let $\mathcal{T}$ be any triangulation of $P$. Then every face $F(I)$ of $P$ is subdivided into $t$ simplices, where $t=n-|I|$. For example we can take the $V$-triangulation of Talman and Yamamoto [24]. Since $\mathcal{T}$ is finite and $P$ is compact, every facet $\tau$ of an $(n-|I|)$ simplex $\sigma$ in $F(I)$ either lies in the boundary of $F(I)$ and is only a facet of $\sigma$ or is a facet of exactly one other $(n-|I|)$-simplex in $F(I)$. Let $f$ be a simplicial approximation of $\varphi$ with respect to $\mathcal{T}$. This means that $f(x) \in \varphi(x)$ for each vertex of $\mathcal{T}$ and $f$ is affine on each simplex of $\mathcal{T}$.

A row vector is lexicopositive if it is a nonzero vector and its first nonzero entry is positive. A matrix is said to be lexicopositive if all its rows are lexicopositive. A matrix is said to be semilexicopositive if each row except possibly the last row is lexicopositive.

Definition 4.1. Let $\tau\left(x^{1}, \ldots, x^{t}\right)$ be a $(t-1)$-simplex in $F(I)$, where $I \in \mathcal{I}$ with $I=\left\{i_{t+1}, \ldots, i_{n}\right\}, t=n-|I|$. The $(n+1) \times(n+1)$ matrix

$$
A_{\tau, I}=\left[\begin{array}{ccccccc}
1 & \cdots & 1 & 0 & \cdots & 0 & 0 \\
-f\left(x^{1}\right) & \cdots & -f\left(x^{t}\right) & a^{i_{t+1}} & \cdots & a^{i_{n}} & c
\end{array}\right]
$$

is the label matrix of $\tau$ with respect to $I$. The simplex $\tau$ is I-complete if $A_{\tau, I}^{-1}$ exists and is semilexicopositive; i.e., the first nonzero entry in every row, except possibly the last one, is positive.

Notice that if for an $I$-complete simplex $\tau$ we change the ordering of the first $n$ columns of the matrix $A_{\tau, I}$, the inverse of the resulting matrix still exists and is semilexicopositive. Clearly, if, for some $I \in \mathcal{I}$, a $(t-1)$-simplex $\tau\left(x^{1}, \ldots, x^{t}\right)$ is an $I$-complete facet of a simplex $\sigma\left(x^{1}, \ldots, x^{t+1}\right)$ in some face $F(I)$, then the system of $n+1$ linear equations with $n+2$ variables

$$
\sum_{j=1}^{t+1} \lambda_{j}\left(\begin{array}{c}
1 \\
-f\left(x^{j}\right)
\end{array}\right)+\sum_{i \in I} \mu_{i}\left(\begin{array}{c}
0 \\
a^{i}
\end{array}\right)+\beta\left(\begin{array}{l}
0 \\
c
\end{array}\right)=\left(\begin{array}{c}
1 \\
0^{n}
\end{array}\right)
$$

has a solution $(\lambda, \mu, \beta)=\left(\lambda_{1}, \ldots, \lambda_{t+1},\left(\mu_{i}\right)_{i \in I}, \beta\right)$ satisfying $\lambda_{j} \geq 0$ for $j \in I_{t+1}$ and $\mu_{i} \geq 0$ for $i \in I$, with $\lambda_{t+1}=0$. Let $x$ be defined by $x=\sum_{j=1}^{t+1} \lambda_{j} x^{j}$ at a solution $(\lambda, \mu, \beta)$ of $(*)$; then $x$ lies in $\sigma$ and is a parametrized stationary point of $f$ with respect to $c$.

The following result is a special case of Theorem 2.6 in Fujishige and Yang [10] and will be used later. This result has been proved in a constructive way.

TheOREM 4.2. Consider any polytope $\mathrm{Q}$ given by $\mathrm{Q}=\left\{x \in \mathbb{R}^{n} \mid c^{i \top} x \leq d_{i}, i \in\right.$ $I_{n}$ and $\left.c^{0 \top} x=d_{0}\right\}$. Assume that $\mathrm{Q}$ is an $(n-1)$-dimensional simple polytope with no redundant constraints. For any $g \in \mathbb{R}^{n}$, there exists a unique subset $I=\left\{j_{1}, \ldots, j_{n-1}\right\}$ 
of $I_{n}$ with $|I|=n-1$ such that the matrix

$$
B^{-1}=\left[\begin{array}{cccccc}
1 & 0 & 0 & \cdots & 0 & 0 \\
g & c^{j_{1}} & c^{j_{2}} & \cdots & c^{j_{n-1}} & c^{0}
\end{array}\right]^{-1}
$$

exists and is semilexicopositive.

We now show that each of the two 0 -simplices $\left\{x^{-}\right\}$and $\left\{x^{+}\right\}$is $I$-complete in the face $F(I)$ for a unique index set $I \in \mathcal{I}$ containing $n-1$ indices.

Lemma 4.3. Let $x^{1}=x^{-}$and $\tau=\left\{x^{1}\right\}$. Then there exists a unique subset $I=\left\{j_{1}, \ldots, j_{n-1}\right\}$ of $I^{-}$with $|I|=n-1$ such that $\tau$ is an I-complete 0 -simplex in $F(I)$.

Proof. Recall that $x^{-}$is a unique solution to the problem

$$
\min c^{\top} x \text { s.t. } x \in P \text {. }
$$

By duality theory there exists a unique solution $\lambda_{i}>0 \forall i \in I^{-}$such that $-c=$ $\sum_{i \in I^{-}} \lambda_{i} a^{i}$. In other words, the vectors $c$ and $a^{i}, i \in I^{-}$, are affinely independent. Consider the following polyhedron:

$$
W=\left\{x \in \mathbb{R}^{n} \mid a^{i \top} x \leq 1, i \in I^{-}, c^{\top} x \leq 1\right\} .
$$

It is easy to see that $W$ is bounded and contains $0^{n}$ in its interior and therefore is an $n$-dimensional polytope. Then the set

$$
Q=\left\{x \in \mathbb{R}^{n} \mid a^{i \top} x \leq 1, i \in I^{-}, c^{\top} x=1\right\}
$$

is an $(n-1)$-dimensional polytope. Let $g=-f\left(x^{-}\right)$. Now all the conditions of Theorem 4.2 are satisfied. So there exists a unique subset $I=\left\{j_{1}, \ldots, j_{n-1}\right\}$ of $I^{-}$ with $|I|=n-1$ such that the matrix

$$
B^{-1}=\left[\begin{array}{cccccc}
1 & 0 & 0 & \cdots & 0 & 0 \\
-f\left(x^{-}\right) & a^{j_{1}} & a^{j_{2}} & \cdots & a^{j_{n-1}} & c
\end{array}\right]^{-1}
$$

exists and is semilexicopositive. This means that $\tau$ is $I$-complete. Clearly, $\tau$ lies in $F(I)$ since $F\left(I^{-}\right)$is a subset of $F(I)$ and $\tau=F\left(I^{-}\right)$.

LEMmA 4.4. Let $x^{1}=x^{+}$and $\tau=\left\{x^{1}\right\}$. Then there exists a unique subset $I=\left\{j_{1}, \ldots, j_{n-1}\right\}$ of $I^{+}$with $|I|=n-1$ such that $\tau$ is an I-complete 0 -simplex in $F(I)$.

Proof. Notice that $x^{+}$is the unique solution to the problem

$$
\max c^{\top} x \text { s.t. } x \in P \text {. }
$$

By duality theory there exists a unique solution $\lambda_{i}>0 \forall i \in I^{+}$such that $c=$ $\sum_{i \in I^{+}} \lambda_{i} a^{i}$. In other words, the vectors $-c$ and $a^{i}, i \in I^{+}$, are affinely independent. Consider the following polyhedron:

$$
W=\left\{x \in \mathbb{R}^{n} \mid a^{i \top} x \leq 1, i \in I^{+},-c^{\top} x \leq 1\right\} .
$$

It is easy to see that $W$ is an $n$-dimensional polytope. Then the set

$$
Q=\left\{x \in \mathbb{R}^{n} \mid a^{i \top} x \leq 1, i \in I^{+}, c^{\top} x=-1\right\}
$$


is an $(n-1)$-dimensional polytope. Let $g=-f\left(x^{+}\right)$. Now all the conditions of Theorem 4.2 are satisfied. So there exists a unique subset $I=\left\{j_{1}, \ldots, j_{n-1}\right\}$ of $I^{+}$ with $|I|=n-1$ such that the matrix

$$
B^{-1}=\left[\begin{array}{cccccc}
1 & 0 & 0 & \cdots & 0 & 0 \\
-f\left(x^{+}\right) & a^{j_{1}} & a^{j_{2}} & \cdots & a^{j_{n-1}} & c
\end{array}\right]^{-1}
$$

exists and is semilexicopositive. This means that $\tau$ is $I$-complete. Clearly, $\tau$ lies in $F(I)$ since $F\left(I^{+}\right)$is a subset of $F(I)$ and $\tau$ lies in $F\left(I^{+}\right)$.

The following lemma is well known in linear programming theory and can easily be proved. We will invoke it later. Let $B$ be a matrix. We denote its $i$ th row by $B_{i}$. and its $j$ th column by $B_{. j}$.

Lemma 4.5. Let $B=\left(B_{.1}, \ldots, B_{. n+1}\right)$ be any nonsingular $(n+1) \times(n+1)$ matrix and let $x$ be any vector in $\mathbb{R}^{n+1}$. Let $k \in I_{n+1}$ and

$$
\bar{B}=\left(B_{.1}, \ldots, B_{. k-1}, x, B_{. k+1}, \ldots, B_{. n+1}\right) .
$$

Then either $\left(B^{-1} x\right)_{k}=0$ and $\bar{B}$ is singular, or $\left(B^{-1} x\right)_{k} \neq 0, \bar{B}$ is nonsingular, and $\bar{B}^{-1}$ is given by

$$
\bar{B}^{-1}=\left[\begin{array}{c}
\left(B^{-1}\right)_{1 .}-\frac{\left(B^{-1} x\right)_{1}}{\left(B^{-1} x\right)_{k}}\left(B^{-1}\right)_{k} \\
\vdots \\
\left(B^{-1}\right)_{k-1 .}-\frac{\left(B^{-1} x\right)_{k-1}}{\left(B^{-1} x\right)_{k}}\left(B^{-1}\right)_{k} \\
\frac{1}{\left(B^{-1} x\right)_{k}}\left(B^{-1}\right)_{k} \\
\left(B^{-1}\right)_{k+1 .}-\frac{\left(B^{-1} x\right)_{k+1}}{\left(B^{-1} x\right)_{k}}\left(B^{-1}\right)_{k} \\
\vdots \\
\left(B^{-1}\right)_{n+1 .}-\frac{\left(B^{-1} x\right)_{n+1}}{\left(B^{-1} x\right)_{k}}\left(B^{-1}\right)_{k}
\end{array}\right]
$$

Lemma 4.6. Let $\sigma$ be a t-simplex in $F(I)$, where $I \in \mathcal{I}, t=n-|I|$, and $I=\left\{i_{t+1}, \ldots, i_{n}\right\}$. If $\sigma$ has an $I$-complete facet $\tau$, then exactly one of the following two cases occurs:

(1) The simplex $\sigma$ is an $\bar{I}$-complete simplex in $F(\bar{I})$, where $\bar{I}=I \backslash\{i\}$ for precisely one index $i \in I$.

(2) The simplex $\sigma$ has exactly one other $I$-complete facet $\bar{\tau}$.

Proof. Let $x^{t+1}$ be the vertex of $\sigma$ opposite to $\tau$, and let $y=A_{\tau, I}^{-1}\left(1,-f\left(x^{t+1}\right)^{\top}\right)^{\top}$. Notice that $y \neq 0^{n+1}$. Let $K=\left\{i \in I_{n} \mid y_{i}>0\right\}$. We first prove $|K|>0$. Since $A_{\tau, I} y=\left(1,-f\left(x^{t+1}\right)^{\top}\right)^{\top}$, we have $\sum_{i=1}^{t} y_{i}=1$. This implies that there exists at least one index $i \in I_{t}$ such that $y_{i}>0$. Hence $K$ is nonempty.

Consider the ratio vectors $\left(1 / y_{j}\right)\left(A_{\tau, I}^{-1}\right)_{j} . \forall j \in K$. Choose $k \in K$ such that the $k$ th ratio vector is the minimum in the lexicographic order over all such ratio vectors. Since $A_{\tau, I}^{-1}$ is regular, $k$ is uniquely determined. Now, we consider the following two cases.

(1) If $k \in I_{n} \backslash I_{t}$, then let $l=i_{k}$ and $\bar{I}=I \backslash\{l\}$. Clearly, $\bar{I} \in \mathcal{I}$ and $\sigma$ is in $F(\bar{I})$. Let $B$ be the matrix obtained from $A_{\tau, I}$ by replacing its $k$ th column by $\left(1,-f\left(x^{t+1}\right)^{\top}\right)^{\top}$. It follows from Lemma 4.5 that $B^{-1}$ exists and is semilexicopositive. By reordering the columns of $B$ we get $A_{\sigma, \bar{I}}$ whose inverse exists and is semilexicopositive. So $\sigma$ is $\bar{I}$-complete. 
(2) If $k \in I_{t}$, then let $\bar{\tau}$ be the facet of $\sigma$ opposite to the vertex $x^{k}$. Using Lemma 4.5, it follows from the choice of $k$ that $A_{\bar{\tau}, I}^{-1}$ exists and is semilexicopositive. Hence $\bar{\tau}$ is an $I$-complete $(t-1)$-simplex in $F(I)$.

It follows immediately from Lemma 4.5 that if any column other than the $k$ th column is replaced, then the inverse of the resulting matrix is not semilexicopositive.

In Lemma 4.8 we consider the analogous case of making a lexicographic pivot step with a column $\left(1, a^{i \top}\right)^{\top}$. First we need the next lemma.

Lemma 4.7. For any set $I \in \mathcal{I}$ with $I \neq I^{-}$and $I \neq I^{+}$, there exist no solutions $\lambda_{0}, \lambda_{i}, i \in I$, to $\sum_{i \in I} \lambda_{i} a^{i}=\lambda_{0} c$ such that $\lambda_{i} \leq 0 \forall i \in I$ and $\sum_{i \in I} \lambda_{i}<0$.

Proof. We need to consider the following three cases:

Case (1). If $\lambda_{0}=0$, then $\sum_{i \in I} \lambda_{i} a^{i}=\lambda_{0} c=0^{n}$ contradicts the fact that all vectors $a^{i}, i \in I$, are linearly independent.

Case (2). If $\lambda_{0}<0$, then by duality theory $\sum_{i \in I} \lambda_{i} a^{i}=\lambda_{0} c$ and $I \neq I^{+}$contradicts the fact that

$$
c^{\top} x^{+}=\max _{x \in P} c^{\top} x .
$$

Case (3). If $\lambda_{0}>0$, then by duality theory $\sum_{i \in I} \lambda_{i} a^{i}=\lambda_{0} c$ and $I \neq I^{-}$contradicts the fact that

$$
c^{\top} x^{-}=\min _{x \in P} c^{\top} x .
$$

Lemma 4.8. Let $\sigma$ be an $I$-complete $(t-1)$-simplex in $F(I)$, where $I \in \mathcal{I}$, $t=n-|I|$, and $I=\left\{i_{t+1}, \ldots, i_{n}\right\}$. If $\sigma$ is in $F(\bar{I})$ and $\bar{I} \neq I^{-}$or $\bar{I} \neq I^{+}$, where $\bar{I}=I \cup\{l\} \in \mathcal{I}$ for some $l \in I_{m} \backslash I$, then exactly one of the following two cases occurs:

(1) There exists a unique set $J \in \mathcal{I}$ with $|J|=|I|$ and $J \neq I$ so that $\sigma$ is in $F(J)$ and is $J$-complete.

(2) There exists exactly one facet $\tau$ of $\sigma$ which is in $F(\bar{I})$ and is $\bar{I}$-complete.

Proof. Let $x=\left(0, a^{l \top}\right)^{\top}$ and $y=A_{\sigma, I}^{-1} x$. Note that $y \neq 0^{n+1}$. Let $K=\left\{i \in I_{n} \mid\right.$ $\left.y_{i}>0\right\}$. Note that $A_{\sigma, I} y=\left(0, a^{l \top}\right)^{\top}$. We need to consider the following two cases.

Case (i). If there exists an index $j \in I_{t}$ such that $y_{j}<0$, then there must exist an index $i \in I_{t}$ such that $y_{i}>0$ since $\sum_{k=1}^{t} y_{i}=0$. Hence $K$ is nonempty.

Case (ii). Suppose that $y_{i}=0 \forall i \in I_{t}$. If $y_{i} \leq 0 \forall i=t+1, t+2, \ldots, n$, then we have that $a^{l}=\sum_{i=t+1}^{n} y_{i} a^{j_{i-t}}+y_{n+1} c$. By Lemma 4.7 it is impossible since $\bar{I}$ is neither equal to $I^{-}$nor equal to $I^{+}$. Hence there exists at least one index $i \in I_{n} \backslash I_{t}$ such that $y_{i}>0$. Again $K$ is nonempty.

Consider the ratio vectors $\left(1 / y_{j}\right)\left(A_{\sigma, I}^{-1}\right)_{j} \forall j \in K$. Choose $k \in K$ such that the $k$ th ratio vector is the minimum in the lexicographic order over all such ratio vectors. Since $A_{\tau, I}^{-1}$ is regular, $k$ is uniquely determined. Now, we consider the following two cases.

(1) If $k \in I_{n} \backslash I_{t}$, then let $p=i_{k}$ and $J=I \cup\{l\} \backslash\{p\}$. Clearly, $J \in \mathcal{I}, J \neq I$, $|J|=|I|$, and $\sigma$ is in $F(J)$. Let $B$ be the matrix obtained from $A_{\sigma, I}$ by replacing its $k$ th column by $x$. It follows from Lemma 4.5 that $B^{-1}$ exists and is semilexicopositive. It is clear that $A_{\sigma, J}=B$. Thus $\sigma$ is a $J$-complete $(t-1)$-simplex in $F(J)$.

(2) If $k \in I_{t}$, then let $\tau$ be the facet of $\sigma$ opposite to the vertex $x^{k}$. Clearly, $\tau$ is a $(t-2)$-simplex in $F(\bar{I})$. Let $B$ be the matrix obtained from $A_{\sigma, I}$ by replacing its $k$ th column by $x$. It follows from Lemma 4.5 that $B^{-1}$ exists and is semilexicopositive. By reordering the columns of $B$ we get $A_{\tau, \bar{I}}$, whose inverse also exists and is semilexicopositive. So $\tau$ is an $\bar{I}$-complete $(t-2)$-simplex in $F(\bar{I})$. 
Again it follows from Lemma 4.5 that if any other column is replaced, then the new matrix is no longer semilexicopositive.

We construct a graph $G=(\mathcal{V}, \mathcal{A})$, where $\mathcal{V}$ denotes the set of nodes and $\mathcal{A}$ denotes the set of edges. Each $I$-complete $(n-|I|-1)$-simplex is a node in $\mathcal{V}$. An $I$-complete $(n-|I|-1)$-simplex $\tau^{1}$ in $F(I)$ and a $J$-complete $(n-|J|-1)$-simplex $\tau^{2}$ in $F(J)$ are said to be adjacent complete simplices if $I=J=L$ and $\tau^{1}$ and $\tau^{2}$ are both facets of an $(n-|L|)$-simplex $\sigma$ in $F(L)$, or $\tau^{1}$ is a facet of $\tau^{2}$ and $\tau^{2}$ is an $(n-|I|)$-simplex in $F(I)$, or $\tau^{2}$ is a facet of $\tau^{1}$ and $\tau^{1}$ is an $(n-|J|)$-simplex in $F(J)$. Two adjacent complete simplices $\tau^{1}$ and $\tau^{2}$ are connected by an edge $e=\left\{\tau^{1}, \tau^{2}\right\} \in \mathcal{A}$. The degree of a node $\tau$ in $G$ is defined to be the number of nodes connected with it, denoted by $\operatorname{deg}(\tau)$. A path in $G$ from node $\tau^{0}=\left\{x^{-}\right\}$to node $\tau^{l}$ is defined as a sequence of the form $\left(\tau^{0}, e_{1}, \tau^{1}, \ldots, e_{l}, \tau^{l}\right)$, where $\tau^{0}, \tau^{1}, \ldots, \tau^{l}$ are nodes and $e_{1}, \ldots, e_{l}$ are edges such that $e_{i}=\left\{\tau^{i-1}, \tau^{i}\right\}$ for $i \in I_{l}$. A path is simple if all its nodes and edges are different.

TheOREm 4.9. Let $\mathcal{T}$ be a triangulation of $P$. Starting at the vertex $x^{-}$, the algorithm generates a finite sequence of adjacent $J$-complete simplices for varying $J \in \mathcal{I}$ which leads to the vertex $x^{+}$.

Proof. By Lemma 4.6, $\left\{x^{-}\right\}$is an $I$-complete 0-simplex in $F(I)$ for some unique set $I \in \mathcal{I}$ with $|I|=n-1$. Since $\left\{x^{-}\right\}$lies in the boundary of $F(I)$, there exists a unique 1-simplex $\sigma$ in $F(I)$ having $\left\{x^{-}\right\}$as its facet. By Lemma 4.6, either $\sigma$ is an $\bar{I}$-complete simplex in $F(\bar{I})$, where $\bar{I}=I \backslash\{i\}$ for some unique $i \in I$, or $\sigma$ has exactly one other $I$-complete facet $\bar{\tau}$. Hence there exists a unique adjacent complete simplex to $\left\{x^{-}\right\}$. That is, $\operatorname{deg}\left(\left\{x^{-}\right\}\right)=1$. Similarly, by using Lemmas 4.6 and 4.8 , we can prove $\operatorname{deg}\left(\left\{x^{+}\right\}\right)=1$.

In all other cases, we prove that if $\tau$ is an $I$-complete $(n-|I|-1)$-simplex in $F(I)$ for some $I \in \mathcal{I}, \tau$ has exactly two adjacent complete simplices. There are two possibilities: either $\tau$ lies in the interior of $F(I)$ or $\tau$ lies in the boundary of $F(I)$. If $\tau$ lies in the interior of $F(I)$, then $\tau$ is a facet of exactly two $(n-|I|)$-simplices in $F(I)$. It follows from Lemma 4.8 that $\tau$ is adjacent to exactly two complete simplices. If $\tau$ lies in the boundary of $F(I)$, then there exists exactly one $(n-|I|)$-simplex $\sigma$ in $F(I)$ having $\tau$ as its facet. By Lemma 4.8 either $\sigma$ is an $\bar{I}$-complete $(n-|\bar{I}|-1)$-simplex in $F(\bar{I})$ for some unique $\bar{I} \in \mathcal{I}$ with $|\bar{I}|=|I|-1$ and has no other $I$-complete facets, or $\sigma$ has exactly one other $I$-complete facet. This yields one adjacent complete simplex to $\tau$. On the other hand, since $\tau$ lies in the boundary of $F(I), \tau$ lies in $F(\tilde{I})$ for some unique set $\tilde{I} \in \mathcal{I}$ with $|\tilde{I}|=|I|+1$. By Lemma 4.8 either $\tau$ is $J$-complete for some unique set $J \in \mathcal{I}$ with $|J|=|I|$ and $J \neq I$, or $\tau$ has exactly one $\tilde{I}$-complete facet. In the former case, $\tau$ lies in $F(\tilde{I})$ and hence there exists exactly one simplex $\bar{\sigma}$ in $F(\tilde{I})$ having $\tau$ as its facet. It follows again from Lemma 4.8 that there exists exactly one other complete simplex adjacent to $\tau$. This concludes that $\tau$ has exactly two adjacent complete simplices. In other words, we have $\operatorname{deg}(\tau)=2$.

As shown above, the degree of each node in the graph $G=(\mathcal{V}, \mathcal{A})$ is at most two. Exactly two nodes have degree equal to one. Since the number of simplices in $P$ is finite, the number of nodes in $G$ must be finite, too. Since $\operatorname{deg}\left(\left\{x^{-}\right\}\right)=1$, it is easy to see that there exists a simple finite path starting from $\left\{x^{-}\right\}$. The end node of this path must be a node $\tau$ of degree one and different from $x^{-}$. The only possibility is that $\tau$ is equal to $\left\{x^{+}\right\}$.

In what follows, if a column of $A_{\tau, I}$ corresponds to a vertex $x^{i}$, we call it a vertex column; if it corresponds to a constraint vector $a^{j}$, we call it an index column. Now we summarize the steps of the algorithm. 


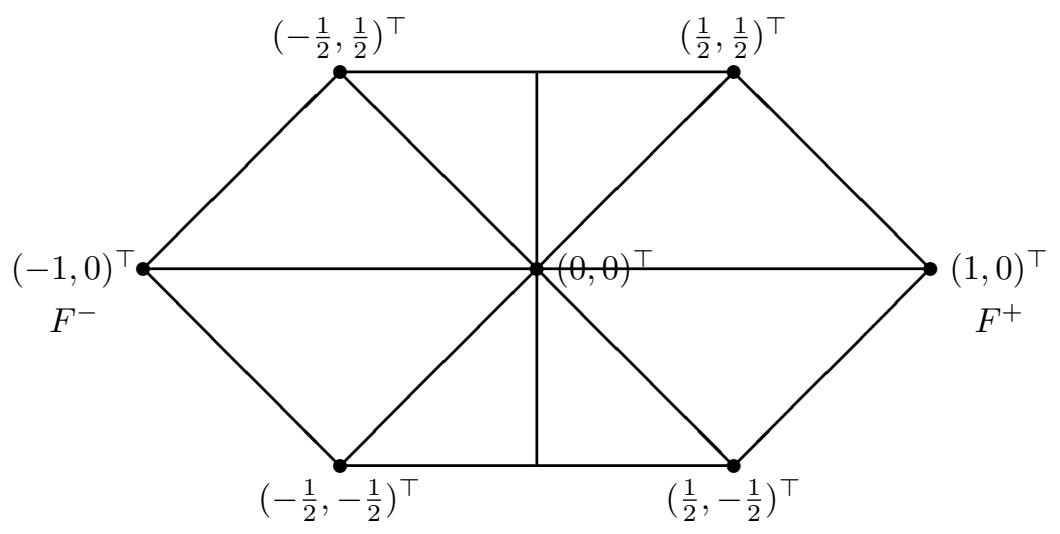

FiG. 4.1. The triangulation of $P$ in Example 3.1 underlying the algorithm.

ALGORITHM.

Step 0 : Take any triangulation of the polytope $P$. Let $j:=0, t:=1, x^{1}:=x^{-}, \tau^{j}:=$ $\tau\left(x^{1}\right)$, and let $x^{t+1}$ be the unique vertex of the simplex in $F(I)$ having $\tau^{j}$ as a facet opposite to it, where $I$ is a set with $n-1$ elements as determined in Lemma 4.3. Go to Step 1.

Step 1 : Let $\sigma$ be equal to the convex hull of $x^{t+1}$ and $\tau^{j}$. Pivot $\left(1,-f\left(x^{t+1}\right)^{\top}\right)^{\top}$ lexicographically into matrix $A_{\tau^{j}, I}$. As described in Lemma 4.6, a unique column $k$ from the first $n$ columns of $A_{\tau^{j}, I}$ will be replaced. If the column $k$ is an index column, then go to Step 3. Otherwise, go to Step 2.

Step 2 : Set $j:=j+1$ and let $\tau^{j}$ be the facet of $\sigma$ opposite the vertex $x^{k}$. If $\tau^{j}=\left\langle x^{+}\right\rangle$, then the algorithm terminates. If $\tau^{j}$ lies in $F(\bar{I})$, where $\bar{I}=I \cup\{l\}$ for some $l \in I_{m} \backslash I$, then go to Step 4. Otherwise there is exactly another simplex $\widetilde{\sigma}$ in $F(I)$ having $\tau^{j}$ as its facet. Go to Step 1 with $x^{t+1}$ as the unique vertex in $\widetilde{\sigma}$ opposite to the facet $\tau^{j}$.

Step 3 : Let $i \in I$ be the index corresponding to the column $k$. Set $I:=I \backslash\{i\}$. There is a unique simplex $\widetilde{\sigma}$ in $F(I)$ having $\sigma$ as a facet. Set $j:=j+1, t:=t+1$, and go to Step 1 with $x^{t+1}$ as the unique vertex in $\widetilde{\sigma}$ opposite to $\sigma$, and $\tau^{j}:=\sigma$.

Step 4 : Set $\sigma:=\tau^{j}$. Pivot $\left(0, a^{l \top}\right)^{\top}$ lexicographically into matrix $A_{\tau^{j}, I}$. By Lemma 4.8 there is a unique column $k$ of the first $n$ columns of $A_{\tau^{j}, I}$ which has to be replaced. If the column $k$ is an index column, then go to Step 3. Otherwise go to Step 2 with $t:=t-1$ and $I:=\bar{I}$.

It is worth mentioning that the algorithm can also start with the simplex $\left\langle x^{+}\right\rangle$ and terminates with the simplex $\left\langle x^{-}\right\rangle$. Following the above description, it is fairly easy to implement the algorithm on a computer.

From Theorem 4.9 and the system of equations $(*)$ we see that every simplex from $\left\langle x^{-}\right\rangle$to $\left\langle x^{+}\right\rangle$contains a parametrized stationary point of the piecewise linear approximation $f$ of $\varphi$ with respect to $\mathcal{T}$. By taking the straight line segments between the parametrized stationary points of any two adjacent simplices, we obtain a piecewise linear path of parametrized stationary points of $f$ connecting the points $x^{-}$and $x^{+}$.

COROLlary 4.10. Let $\mathcal{T}$ be any triangulation of $P$. Then with respect to the vector $c$ there is a piecewise linear path of parametrized stationary points of the piecewise linear approximation $f$ of $\varphi$ with respect to $\mathcal{T}$ and this path connects $x^{-}$ 


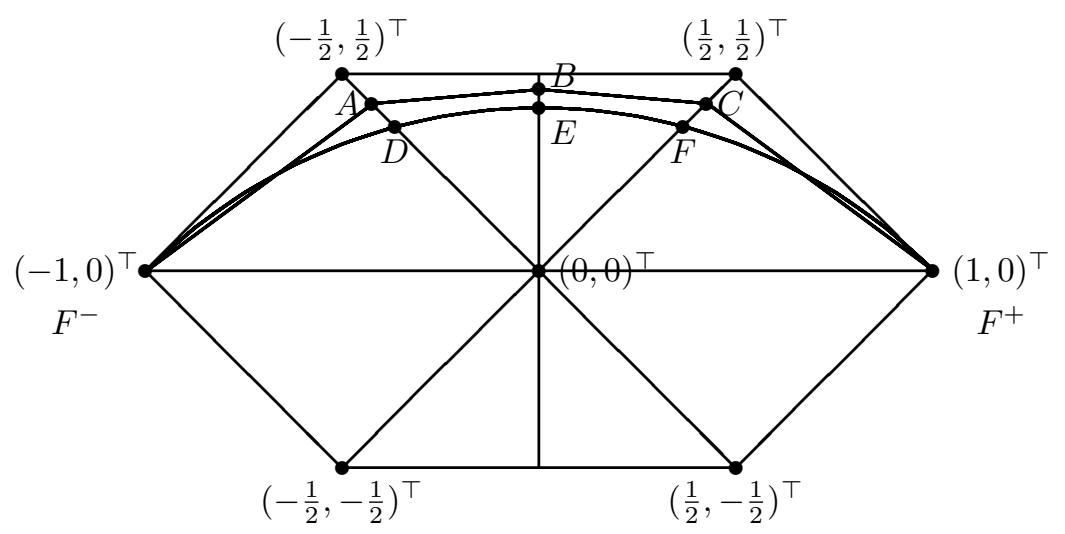

FIG. 4.2. The piecewise linear path generated by the algorithm in Example 3.1.

and $x^{+}$.

Now we will illustrate the algorithm with Example 3.1. To implement the algorithm, we take the triangulation depicted in Figure 4.1. Note that this triangulation is very coarse. The piecewise linear path generated by the algorithm is given by the sequence of line segments $\left[(-1,0)^{\top},(-a, a)^{\top}\right],\left[(-a, a)^{\top},(0, b)^{\top}\right],\left[(0, b)^{\top},(a, a)^{\top}\right]$, and $\left[(a, a)^{\top},(1,0)^{\top}\right]$, where $a=(5-\sqrt{7})^{-1}$ and $b=(5-2 \sqrt{2})^{-1}$; see Figure 4.2. As we see from Figure 4.2 the path of approximate zero points generated by the algorithm and the set of the actual zero points of $f$ are quite close. The finer the triangulation is the more accurate the approximation will be. In this figure $A=(-a, a)^{\top}, B=(0, b)^{\top}$, $C=(a, a)^{\top}, D=(-d, d)^{\top}, E=(0, e)^{\top}$, and $F=(d, d)^{\top}$ with $d=\sqrt{3} / 2-1 / 2$ and $e=\sqrt{2}-1$.

5. Proofs for the existence theorems. In this section we still assume that $x^{-}$ and $x^{+}$are unique. First it will be argued in Theorems 5.1 and 5.2 that the points lying on the path given in Corollary 4.10 indeed all correspond to approximate parametrized stationary or zero points of the mapping $\varphi$. To show this, a sequence of triangulations $\mathcal{T}^{r}$ with mesh size converging to zero is taken. This yields, according to Corollary 4.10, for every $r \in \mathbb{N}$, a continuous piecewise linear function $\pi^{r}:[0,1] \rightarrow P$ with image set $\pi^{r}([0,1])$ connecting $x^{-}$and $x^{+}$. It will be shown that if $q^{r}$ is an arbitrary point in $\pi^{r}([0,1])$ and the sequence $\left(q^{r}\right)_{r \in \mathbb{N}}$ converges to $q$, then $q$ is a parametrized stationary point of $\varphi(q)$ with respect to $c$. Under the conditions of Theorems 3.2, 3.3, and 3.4, the piecewise linear approximation can be chosen in such a way that $q$ is a zero point of $\varphi$. Furthermore, it will be shown in Theorem 5.4 by a limiting argument that there exists a connected set of zero points of $\varphi$, containing both $x^{-}$and $x^{+}$, that is being approximated.

TheOREm 5.1. Let $\varphi: P \Longrightarrow \mathbb{R}^{n}$ be a correspondence satisfying Assumption 2.1 and let $c \in \mathbb{R}^{n} \backslash\left\{0^{n}\right\}$. For $r \in \mathbb{N}$, let $\mathcal{T}^{r}$ be a triangulation of $P$ with mesh size smaller than $\frac{1}{r}$, let $f^{r}: P \rightarrow \mathbb{R}^{n}$ be a piecewise linear approximation of $\varphi$ with respect to $\mathcal{T}^{r}$, and let $\pi^{r}:[0,1] \rightarrow P$ be the corresponding continuous function with image set connecting $x^{-}$and $x^{+}$. Let $\left(q^{r}\right)_{r \in \mathbb{N}}$ be an arbitrary convergent sequence of points in $P$ with limit $q^{*}$ where $q^{r} \in \pi^{r}([0,1])$. Then $q^{*}$ is a parametrized stationary point of $\varphi$ 
with respect to $c$.

Proof. Let $\left(\lambda_{1}^{r}, \ldots, \lambda_{n+1}^{r}, x^{1^{r}}, \ldots, x^{n+1^{r}}, s^{1^{r}}, \ldots, s^{n+1^{r}}\right)_{r \in \mathbb{N}}$ be a sequence of points in $\mathbb{R}_{+}^{n+1} \times \prod_{i=1}^{n+1} P \times \prod_{i=1}^{n+1} \mathbb{R}^{n}$ satisfying that $\sum_{j=1}^{n+1} \lambda_{j}^{r}=1, \sigma^{r}\left(x^{1^{r}}, \ldots, x^{n+1^{r}}\right)$ is a simplex of $\mathcal{T}^{r}, q^{r}=\sum_{j=1}^{n+1} \lambda_{j}^{r} x^{j^{r}} \in \pi^{r}([0,1])$, and $s^{j^{r}}=f^{r}\left(x^{j^{r}}\right)$. Notice that it may happen that $\lambda_{j}^{r}=0$ for some $j \in I_{n+1}$. By definition, $f^{r}\left(q^{r}\right)=\sum_{j=1}^{n+1} \lambda_{j}^{r} s^{j^{r}}$. Define $s^{r}=f^{r}\left(q^{r}\right)$; then $s^{r}=\beta^{r} c+\sum_{j \in I^{r}} \mu_{j}^{r} a^{j}$ for some $\beta^{r} \in \mathbb{R}$ for some $\mu_{j}^{r} \geq 0 \forall j \in I^{r}$, and for some $I^{r} \in \mathcal{I}$ satisfying that $q^{r}$ lies in $F\left(I^{r}\right)$. Since $\cup_{q \in P} \varphi(q)$ is bounded, the sequence given above remains in a compact set, and without loss of generality it can be assumed to converge to an element $\left(\lambda_{1}^{*}, \ldots, \lambda_{n+1}^{*}, x^{* 1}, \ldots, x^{* n+1}, s^{* 1}, \ldots, s^{* n+1}\right)$. Define $s^{*}=\sum_{j=1}^{n+1} \lambda_{j}^{*} s^{* j}$. Clearly, it holds that $s^{r} \rightarrow s^{*}$. Since for every $r \in \mathbb{N}$ the mesh size of $\mathcal{T}^{r}$ is smaller than $\frac{1}{r}$, it holds for every $j \in I_{n+1}$ that $x^{* j}=q^{*}$. Using that $\varphi$ is upper semicontinuous this implies that for every $j \in I_{n+1}, s^{* j} \in \varphi\left(q^{*}\right)$. Moreover, $\beta^{r} \rightarrow \beta^{*}$ for some number $\beta^{*}$, without loss of generality $I^{r}=I^{*} \forall r$ for some $I^{*} \in \mathcal{I}$, and $\mu_{j}^{r} \rightarrow \mu_{j}^{*} \forall j \in I^{*}$ for some nonnegative $\mu_{j}^{*}$. Since $\varphi$ is convex valued, $\sum_{j=1}^{n+1} \lambda_{j}^{*}=1$ and $\lambda_{j}^{*} \geq 0 \forall j \in I_{n+1}$, it holds that $s^{*} \in \varphi\left(q^{*}\right)$. Moreover, $q^{*} \in F\left(I^{*}\right)$ and $s^{*}=\beta^{*} c+\sum_{j \in I^{*}} \mu_{j}^{*} a^{j} \in A\left(I^{*}\right)$. Hence, according to Lemma $2.3, q^{*}$ is a parametrized stationary point of $\varphi$ with respect to $c$.

In order to give a constructive proof of Theorems 3.2, 3.3, and 3.4, the piecewise linear approximation $f$ of $\varphi$ with respect to a triangulation $\mathcal{T}$ should be chosen as follows. We call such a piecewise linear approximation a proper one. In case of Theorem 3.2 any piecewise linear approximation of $\varphi$ with respect to $\mathcal{T}$ can be chosen. Next consider Theorem 3.3. If a point $x$ in the (relative) interior of a face $F(I)$ is a vertex of a simplex of the triangulation, this implies that at least one element in $\varphi(x)$ lies in the set $A_{0}^{*}(I)$, and this element is assigned to the piecewise linear approximation at $x$. In the case of Theorem 3.4 an element of the set $A^{*}(I)$ in $\varphi(x)$ is assigned to a vertex $x$ of a simplex if $x$ lies in (the interior of) $F(I)$.

THEOREM 5.2. Let $\varphi: P \Longrightarrow \mathbb{R}^{n}$ be a correspondence satisfying the conditions in one of Theorems 3.2, 3.3, or 3.4. For $r \in \mathbb{N}$, let $\mathcal{T}^{r}$ be a triangulation of $P$ with mesh size smaller than $\frac{1}{r}$ and let $f^{r}: P \rightarrow \mathbb{R}^{n}$ be a proper piecewise linear approximation of $\varphi$ with respect to $\mathcal{T}^{r}$. Let $\left(q^{r}\right)_{r \in \mathbb{N}}$ be an arbitrary convergent sequence of points in $P$ with limit $q^{*}$ such that for any $r \in \mathbb{N}$ it holds that $q^{r} \in \pi^{r}([0,1])$. Then $q^{*}$ is a zero point of $\varphi$.

Proof. First consider Theorem 3.2. Following the proof of Theorem 5.1 the limit point $s^{*} \in \varphi\left(q^{*}\right)$ is an element of $A\left(I^{*}\right)$, whereas $q^{*}$ is an element of $F\left(I^{*}\right)$. The latter property implies that $s^{*}$ is not an element of $A\left(I^{*}\right)$, unless $s^{*}=0^{n}$. Hence, $s^{*}=0^{n}$. Next consider Theorem 3.3. Consider again the convergent sequence of simplices $\sigma^{r}$ in $F\left(I^{*}\right)$ mentioned in the proof of Theorem 5.1. Then the vertex $x^{j^{r}}$ of $\sigma^{r}$ lies in some face $F\left(I^{j^{r}}\right)$ of $P$ with $I^{*} \subset I^{j^{r}}$. Hence, we have that $A_{0}^{*}\left(I^{j^{r}}\right) \subset A_{0}^{*}\left(I^{*}\right)$, and so $s^{r} \in A_{0}^{*}\left(I^{*}\right) \forall r$ because of the properness of $f^{r}$. Consequently, $s^{*} \in A_{0}^{*}\left(I^{*}\right)$ and therefore $s^{*} \in A_{0}^{*}\left(I^{*}\right) \cap A\left(I^{*}\right) \subset\left\{0^{n}\right\}$, i.e., $s^{*}=0^{n}$. In the case of Theorem 3.4, following a similar argument as in the previous case we obtain that $s^{*} \in A^{*}\left(I^{*}\right) \cap$ $A\left(I^{*}\right)$. Since the latter intersection consists of only the zero vector, we obtain again that $s^{*}=0^{n}$.

From Theorem 5.2 the next result follows immediately.

Corollary 5.3. Let $\varphi: P \Longrightarrow \mathbb{R}^{n}$ be a correspondence satisfying the conditions in one of Theorems 3.2, 3.3, or 3.4. For $r \in \mathbb{N}$, let $\mathcal{T}^{r}$ be a triangulation of $P$ with mesh size smaller than $\frac{1}{r}$ and let $f^{r}: P \rightarrow \mathbb{R}^{n}$ be a proper piecewise linear approximation of $\varphi$ with respect to $\mathcal{T}^{r}$. Then for every $\varepsilon>0$ there exists an $R \in \mathbb{N}$ 
such that for every $r \geq R$ it holds that $q^{r} \in \pi^{r}([0,1])$ implies $\left\|f^{r}\left(q^{r}\right)\right\|_{\infty}<\varepsilon$.

Proof. Suppose that a sequence $\left(q^{r}, f^{r}\left(q^{r}\right)\right)_{r \in \mathbb{N}}$ exists with $q^{r} \in \pi^{r}([0,1])$ and $\left\|f^{r}\left(q^{r}\right)\right\|_{\infty} \geq \varepsilon$ for every $r \in \mathbb{N}$. Since $P$ and $\cup_{q \in P} \varphi(q)$ are compact, there exists a converging subsequence $\left(q^{r^{s}}, f^{r^{s}}\left(q^{r^{s}}\right)\right)_{s \in \mathbb{N}}$, with limit say $\left(q^{*}, s^{*}\right)$, where $\left\|s^{*}\right\|_{\infty} \geq$ $\varepsilon>0$. As in the proof of Theorem 5.2 it can be shown that $s^{*}=0^{n}$, yielding a contradiction.

Using Theorem 4.9 and Theorem 5.2 it will be shown that there exists a connected set $C$ in $P$ such that $x^{-} \in C, x^{+} \in C$, and $0^{n} \in \varphi(q) \forall q \in C$. Hence, there is a continuum of zero points of $\varphi$ being approximated by the algorithm of section 4 . For a nonempty, compact set $S \subset \mathbb{R}^{n}$, define the continuous function $d_{S}: \mathbb{R}^{n} \rightarrow \mathbb{R}$ by $d_{S}(x)=\min \left\{\|x-y\|_{\infty} \mid y \in S\right\}$.

TheOREM 5.4. Let $\varphi: P \Longrightarrow \mathbb{R}^{n}$ be a correspondence satisfying the conditions in one of Theorems 3.2, 3.3, or 3.4. Then there exists a connected set $C$ of points in $P$ such that $x^{-} \in C, x^{+} \in C$, and $0^{n} \in \varphi(q) \forall q \in C$.

Proof. Define $Q=\left\{q \in P \mid 0^{n} \in \varphi(q)\right\}$. From the conditions of the theorems it immediately follows that $x^{-} \in Q, x^{+} \in Q$, and $Q$ is compact. Suppose the theorem is false. Then $x^{+}$is not an element of the component of $Q$ containing $x^{-}$. By Munkres [21, p. 235] it holds for every compact set $X$ in some Euclidean space and for every element $x \in X$ that the component of $X$ containing $x$ equals the intersection of all sets containing $x$ which are both open and closed in $X$. Hence, there exists a set $Q^{0}$, which is open and closed in $Q$, such that $x^{-} \in Q^{0}$ and $x^{+} \notin Q^{0}$. Define $Q^{1}=Q \backslash Q^{0}$. Then $Q^{1}$ is open and closed in $Q, x^{-} \notin Q^{1}$, and $x^{+} \in Q^{1}$. Since $Q$ is compact, it follows that $Q^{0}$ and $Q^{1}$ are disjoint, compact sets. Hence, there exists $\varepsilon>0$ such that $\min \left\{\left\|q^{0}-q^{1}\right\|_{\infty} \mid q^{0} \in Q^{0}, q^{1} \in Q^{1}\right\} \geq \varepsilon$. For every $r \in \mathbb{N}$, let $\mathcal{T}^{r}$ be a triangulation of $P$ with mesh size smaller than $\frac{1}{r}$, let $f^{r}: P \rightarrow \mathbb{R}^{n}$ be a proper piecewise linear approximation of $\varphi$ with respect to $\mathcal{T}^{r}$, and let $\pi^{r}:[0,1] \rightarrow P$ be the corresponding continuous function with image set connecting $x^{-}$and $x^{+}$. Define $g^{r}:[0,1] \rightarrow \mathbb{R}$ by

$$
g^{r}(t)=d_{Q^{0}}\left(\pi^{r}(t)\right)-d_{Q^{1}}\left(\pi^{r}(t)\right) \forall t \in[0,1] .
$$

Since $g^{r}$ is continuous, $g^{r}(0) \leq-\varepsilon$, and $g^{r}(1) \geq \varepsilon$, there exists a point $t^{r} \in[0,1]$ such that $g^{r}\left(t^{r}\right)=0$. Hence, $d_{Q^{0}}\left(\pi^{r}\left(t^{r}\right)\right)=d_{Q^{1}}\left(\pi^{r}\left(t^{r}\right)\right)=d_{Q}\left(\pi^{r}\left(t^{r}\right)\right) \geq \frac{1}{2} \varepsilon$. Without loss of generality, it can be assumed that $\left(\pi^{r}\left(t^{r}\right)\right)_{r \in \mathbb{N}}$ converges to a point $q^{*} \in P$. Hence,

$$
d_{Q}\left(q^{*}\right)=d_{Q}\left(\lim _{r \rightarrow \infty} \pi^{r}\left(t^{r}\right)\right)=\lim _{r \rightarrow \infty} d_{Q}\left(\pi^{r}\left(t^{r}\right)\right) \geq \frac{1}{2} \varepsilon>0 .
$$

However, by Theorem $5.2, d_{Q}\left(q^{*}\right)=0$, yielding a contradiction.

Similarly, one can easily show that in the case of Theorem 3.1 there exists a connected set $C$ of parametrized stationary points in $P$ with respect to $c$ such that $x^{-} \in C$ and $x^{+} \in C$.

6. The general case. The algorithm proposed in the previous section can be adapted for computing a continuum of parametrized stationary points or zero points of $\varphi$ on $P$ in case the faces $F^{-}$and $F^{+}$are not vertices of $P$. First we take any point $v$ in the interior of $F^{-}$and a triangulation of $P$ such that the face $F^{-}$itself is being triangulated according to the $V$-triangulation of Talman and Yamamoto [24]. For $J \in$ $\mathcal{I}$ such that $I^{-} \subset J$, let $V F(J)=\left\{x \in F^{-} \mid x=\lambda v+(1-\lambda) y, 0 \leq \lambda \leq 1, y \in F(J)\right\}$. The $V$-triangulation subdivides any such set $V F(J)$ into $(n-|J|+1)$-simplices.

Then we apply the algorithm of Talman and Yamamoto [24] to find a parametrized stationary point $x^{-}$in $F^{-}$of the piecewise linear approximation $f$ of the correspondence $\varphi$ on $P$ with respect to $c$. To initiate the algorithm, we solve $\max x^{\top} f(v)$ 
subject to $x \in F^{-}$, which yields, by using Theorem 2.6 of Fujishige and Yang [10] for its dual, a uniquely determined vertex $F\left(I_{0}\right)$ of $F^{-}$as a solution, with $\left|I_{0}\right|=n$ and $I^{-} \subset I_{0}$. Then starting with the 0 -simplex $\{v\}$ and $I$ equal to $I_{0}$ the algorithm generates a sequence of adjacent simplices in $V F(I)$ for varying $I \in \mathcal{I}$ such that $I^{-} \subset I$, and the common facets $\tau\left(x^{1}, \ldots, x^{t}\right)$ satisfy that the system of equations

$$
\sum_{j=1}^{t} \lambda_{j}\left(\begin{array}{c}
1 \\
-f\left(x^{j}\right)
\end{array}\right)+\sum_{i \in I} \mu_{i}\left(\begin{array}{c}
0 \\
a^{i}
\end{array}\right)=\left(\begin{array}{c}
1 \\
0^{n}
\end{array}\right)
$$

has a solution $(\lambda, \mu)$ satisfying $\lambda_{j} \geq 0, j \in I_{t}, \mu_{i} \geq 0$ for $i \in I \backslash I^{-}$. Notice that $I^{-} \subset I$ and that we allow $I$ to be equal to $I^{-}$.

The algorithm of Talman and Yamamoto stops as soon as a $(t-1)$-simplex $\tau^{-}\left(x^{1}, \ldots, x^{t}\right)$ in $F(I)$ for some $I$ containing $I^{-}$is generated for which the system has a solution $(\lambda, \mu)$. Then $x=\sum_{j=1}^{t} \lambda_{j} x^{j}$ is a parametrized stationary point in $F\left(I^{-}\right)$ with respect to $c$ of the piecewise linear approximation $f$ of $\varphi$. Next the vector $\left(c^{\top}, 0\right)$ is pivoted semilexicographically into the system, making any $\mu_{i}, i \in I$, nonnegative. Since $-c=\sum_{i \in I^{-}} \lambda_{i} a^{i}$ for unique $\lambda_{i}>0$, one of the $\mu_{i}$ 's, say $\mu_{i_{0}}$, for some $i_{0} \in I^{-}$, will leave the basis. Now the algorithm continues in $F\left(I \backslash\left\{i_{0}\right\}\right)$ with the unique $t$ simplex $\sigma$ in $F\left(I \backslash\left\{i_{0}\right\}\right)$ having $\tau^{-}$as a facet and a semilexicographic pivot step is made with $\left(1,-f^{\top}\left(x^{t+1}\right)\right)^{\top}$ where $x^{t+1}$ is the vertex of $\sigma$ opposite to $\tau$, and so on.

In this way the algorithm generates for varying $I \in \mathcal{I}$ by semilexicographic pivoting a unique sequence of adjacent simplices in $F(I)$ with common $I$-complete facets until a facet $\tau$ being a simplex in $F(H)$ for some $H$ containing $I^{-}$or a complete facet $\tau^{+}$being a simplex in $F(J)$ for some $J$ containing $I^{+}$is generated. In the former case the algorithm continues in the subset $V F(H)$ of $F\left(I^{-}\right)$as above until again an $I$-complete simplex in $F(I)$ for some $I$ containing $I^{-}$is found, and so on. In the latter case the point $x^{+}=\sum_{j=1}^{t} \lambda_{j} x^{j}$ at the solution $(\lambda, \mu, \beta)$ lies in $\tau^{+}$and is a parametrized stationary point in $F^{+}$of $f$ with respect to $c$.

Letting $x^{-}$be the last point being generated in $F^{-}$, the algorithm generates a piecewise linear path of parametrized stationary points of $f$ with respect to the vector $c$. This path connects the point $x^{-}$in the face $F^{-}$with a point $x^{+}$in the face $F^{+}$. Taking a sequence of triangulations of $P$ with mesh tending to zero, in the limit a connected set of parametrized stationary points of $\varphi$ is obtained with respect to $c$ connecting the faces $F^{-}$and $F^{+}$. In case the correspondence $\varphi$ satisfies the conditions of Theorems 3.2, 3.3, or 3.4 and the piecewise linear approximations are chosen in an appropriate way, there exists a connected set of zero points of $\varphi$ connecting $F^{-}$ and $F^{+}$.

Notice that if a sequence of triangulations with mesh tending to zero is taken, for any triangulation in this sequence the points $x^{-}$in $F^{-}$and $x^{+}$in $F^{+}$being connected through the piecewise linear path generated by the algorithm may differ. In the limit these points converge on a subsequence to two different zero points of $\varphi$, one lying in $F^{-}$and the other lying in $F^{+}$.

7. Examples. In this section we will derive three existing existence theorems from Theorems 3.2, 3.3, and 3.4.

The first example is derived from general equilibrium theory with price rigidities. Let $U^{n}=\left\{x \in \mathbb{R}^{n} \mid 0 \leq x_{i} \leq 1 \forall i \in I_{n}\right\}$ be the $n$-dimensional unit cube. Let $1^{n}$ denote the $n$-vector of ones and for $i \in I_{n}$ let $e(i)$ denote the $i$ th unit vector in $\mathbb{R}^{n}$. Under standard assumptions on economic primitives (see, e.g., Herings [12] for details), it can be derived that the total excess demand function of an economy with 
completely fixed prices $p \in \mathbb{R}_{++}^{n}$ is a continuous function $f: U^{n} \rightarrow \mathbb{R}^{n}$ with the following properties:

(A) for every $x \in U^{n}$, for every $j \in I_{n}, x_{j}=0$ implies $f_{j}(x) \geq 0$, and $x_{j}=1$ implies $f_{j}(x) \leq 0$

(B) for every $x \in U^{n}, p^{\top} f(x)=0$.

THEOREM 7.1. Let $f: U^{n} \rightarrow \mathbb{R}^{n}$ be the excess demand function of an economy with completely fixed prices $p \in \mathbb{R}_{++}^{n}$. Then there exists a connected set $C$ of zero points of $f$ such that $0^{n} \in C$ and $1^{n} \in C$.

Proof. We can rewrite the set $U^{n}$ as

$$
U^{n}=\left\{x \in \mathbb{R}^{n} \mid a^{i \top} x \leq b_{i} \quad \forall i \in I_{2 n}\right\},
$$

where $a^{i}=e(i), b_{i}=1, a^{n+i}=-e(i)$, and $b_{n+i}=0 \forall i \in I_{n}$. We can partition any $I \in \mathcal{I}$ into two disjoint subsets $I^{1}$ and $I^{2}$ with $I^{1} \subset I_{n}$ and $I^{2} \subset I_{2 n} \backslash I_{n}$. Notice that $i \in I^{1}$ implies $i+n \notin I^{2}$, and $i \in I^{2}$ implies $i-n \notin I^{1}$.

Let $c=p$. Clearly, $x^{-}=0^{n}$ and $x^{+}=1^{n}$. We check the condition of Theorem 3.2. Suppose $0^{n} \neq f(x) \in A(I)$ for some $I \in \mathcal{I}$, so $f(x)=\sum_{i \in I^{1}} \mu_{i} e(i)-\sum_{i \in I^{2}} \mu_{i} e(i-$ $n)+\beta p$, where $\mu_{i} \geq 0 \forall i \in I$, and $\beta \in \mathbb{R}$, and some $\mu_{i}$ or $\beta$ nonzero. Conditions (A) and (B) imply that $f\left(0^{n}\right)=0^{n}$ and $f\left(1^{n}\right)=0^{n}$, so $I \neq I_{n}$ and $I \neq I_{2 n} \backslash I_{n}$. If $I=\emptyset$, then $0=p^{\top} f(x)=p^{\top} \beta p \neq 0$, a contradiction. Take any nonempty set $I \in \mathcal{I}$ not equal to $I_{n}$ or $I_{2 n} \backslash I_{n}$.

If $I^{1} \neq \emptyset$ and $I^{2} \neq \emptyset$, then $p_{i}\left(\beta+\mu_{i}\right) \leq 0$, for $i \in I^{1}$, and $p_{i-n}\left(\beta-\mu_{i}\right) \geq 0$, for $i \in I^{2}$, so $\beta=0$ and $\mu_{i}=0 \forall i \in I$, a contradiction.

If $I^{1}=\emptyset$, then $I^{2} \neq \emptyset$, so $\beta>0$, but then $f(x)>0^{n}$, a contradiction to $0=p^{\top} f(x)$.

If $I^{2}=\emptyset$, then $I^{1} \neq \emptyset$, so $\beta<0$, but then $f(x)<0^{n}$, a contradiction to $0=p^{\top} f(x)$. Consequently, Theorem 3.2 holds.

The proof of Theorem 7.1 shows that our results apply to economies with completely fixed prices $p \in \mathbb{R}_{++}^{n}$. Since the results apply to all such economies, that is, for all possible specifications of utility functions and initial endowments, it also shows that our results satisfy a certain kind of robustness. The above theorem can also be derived from Theorems 3.3 and 3.4.

The following result is obtained by Herings, Talman, and Yang [15, Theorem 4.3 , p. 690] which generalizes Theorem 7.1. It is therefore also related to economies with price rigidities, and it applies even under somewhat weaker assumptions on the economic primitives. We will show that this theorem is a special case of Theorem 3.3.

TheOREM 7.2. Let $\varphi: U^{n} \Longrightarrow \mathbb{R}^{n}$ be any correspondence satisfying Assumption 2.1. Moreover, it holds that

(A) for every $x \in U^{n}$, there exists $f \in \varphi(x)$ such that, for every $j \in I_{n}, x_{j}=0$ implies $f_{j} \geq 0$, and $x_{j}=1$ implies $f_{j} \leq 0$;

(B) for every $x \in U^{n}$, for every $f \in \varphi(x)$, there exists some $p \in \mathbb{R}_{++}^{n}$ such that $p^{\top} f=0$.

Then there exists a connected set $C$ of zero points of $\varphi$ such that $0^{n} \in C$ and $1^{n} \in C$.

Proof. Rewrite the set $U^{n}$ as in the proof of Theorem 7.1. Let $c=1^{n}$. Clearly, $x^{-}=0^{n}$ and $x^{+}=1^{n}$. Moreover, it is easy to verify that condition (ii) of Theorem 3.3 is satisfied by condition (A). We have to check condition (i). For $I=\emptyset$, condition (i) is trivially satisfied. Now take any nonempty set $I$ from $\mathcal{I}$. We partition $I$ into two disjoint subsets $I^{1}$ and $I^{2}$ as in the proof of Theorem 7.1. Suppose there is some $x \in F(I)$ and some $f \in \varphi(x)$ such that $f \in\left(A_{0}^{*}(I) \cap A(I)\right) \backslash\left\{0^{n}\right\}$. This implies that 
there exist $\mu_{i} \geq 0 \forall i \in I$ and $\beta \in \mathbb{R}$ such that

$$
\begin{aligned}
f & =\sum_{i \in I} \mu_{i} a^{i}+\beta c, \\
f^{\top} a^{i} & \leq 0 \quad \forall i \in I, \\
f & \neq 0^{n} .
\end{aligned}
$$

Equivalently,

$$
\begin{aligned}
f & =\sum_{i \in I^{1}} \mu_{i} e(i)-\sum_{i \in I^{2}} \mu_{i} e(i-n)+\beta c \\
f_{i} & \leq 0 \quad \forall i \in I^{1} \\
f_{i} & \geq 0 \quad \forall n+i \in I^{2} \\
f & \neq 0^{n} .
\end{aligned}
$$

This implies that

$$
\begin{aligned}
& \beta \geq \max _{i \in I^{2}} \mu_{i}, \\
& \beta \leq-\max _{i \in I^{1}} \mu_{i} .
\end{aligned}
$$

In case $I^{2}=\emptyset$, we have $f<0^{n}$. This contradicts condition (B). In case $I^{1}=\emptyset$, we have $f>0^{n}$, again contradicting condition (B). In cases $I^{1} \neq \emptyset$ and $I^{2} \neq \emptyset$, without loss of generality there exist $i \in I^{1}$ and $j \in I^{2}$ such that $\mu_{i}>0$ and $\mu_{j}>0$. This would mean both $\beta>0$ and $\beta<0$ from (7.1) which is impossible. Hence condition (i) is satisfied.

Now we use Theorem 3.4 to show that there is a continuum of constrained equilibria in a pure exchange economy with general price rigidities; see, e.g., Schalk and Talman [23] for details. Price vectors in such an economy with $n$ commodities are restricted to an $n$-dimensional simple polytope

$$
P=\left\{p \in \mathbb{R}_{+}^{n} \mid t^{-} \leq p^{\top} c \leq t^{+}, a^{i^{\top}} p \leq b_{i}, i \in I_{m}\right\}
$$

for some strictly positive vector $c$ with length $1,0<t^{-}<t^{+}, a^{i^{\top}} c=0 \forall i \in I_{m}$. We also assume that there are no redundant constraints and that $P$ is a subset of $\mathbb{R}_{++}^{n}$. We define

$$
Q=\left\{q \in \mathbb{R}^{n} \mid t^{-} \leq q^{\top} c \leq t^{+}, a^{i \top} q \leq b_{i}+\varepsilon \forall i \in I_{m}\right\}
$$

for some $\varepsilon>0$. For $\varepsilon$ small enough, $F(I), I \subset I_{m}$, is a face of $Q$ if and only if $\left\{x \in P \mid a^{i} x=b_{i}, i \in I\right\}$ is a face of $P$. For any $q$ in $Q$, let $p(q)$ be the orthogonal projection of $q$ on $P$ and let $I(q)$ be such that $p(q)$ is in the interior of the face $F(I(q))$ of $P$. Then there exist unique nonnegative numbers $\mu^{i}(q), i \in I(q)$, such that $q=p(q)+\sum_{i \in I(q)} \mu_{i}(q) a^{i}$.

At $q \in Q$ define the price vector by $p(q)$ and a continuous rationing scheme $\left(r^{i}(q), d_{i}(q)\right), i \in I_{m}$, such that $r^{i}(q)=a^{i}, d_{i}(q)=0$ if $\mu_{i}(q)=1$ and $d_{i}(q)=M$ if $\mu_{i}(q)=0$ or $i$ not in $I(q)$, for sufficiently large $M>0$. This rationing scheme determines the constraints on the net-supply of the consumers. Given a utility function $u^{h}$ and initial endowment $w^{h}$, consumer $h \in H$ maximizes his utility $u^{h}(x)$ over his budget constraint given by $p(q)^{\top} x \leq p(q)^{\top} w^{h}$ and rationing constraints $r^{i}(q)^{\top}\left(x-w^{h}\right) \leq d_{i}(q), i \in I_{m}$. The solution set $x^{h}(q)$ yields the constraint excess demand set $z^{h}(q)=x^{h}(q)-\left\{w^{h}\right\}$ of consumer $h \in H$ at $q$. Adding up these sets over all consumers in $H$ gives the total constraint excess demand correspondence $\zeta: Q \rightarrow \mathbb{R}^{n}$. Under certain standard economic conditions, the correspondence $\zeta$ satisfies Assumption 2.1 and $p(q)^{\top} z=0$ for any $z \in \zeta(q), q \in Q$. 
A constrained equilibrium is obtained if $q \in P$ is such that $0^{n} \in \zeta(q)$. At such an equilibrium, $p(q)^{\top} a^{i}=b_{i}$ if the $i$ th rationing scheme is binding.

Let $F^{-}$be the face of $Q$ on which $c^{\top} x$ is minimized on $Q$ and let $F^{+}$be the face of $Q$ on which $c^{\top} x$ is maximized on $Q$. Notice that $F^{-}=\left\{q \in Q \mid q^{\top} c=t^{-}\right\}$and $F^{+}=\left\{q \in Q \mid q^{\top} c=t^{+}\right\}$.

To see that there is a connected set of constrained equilibria linking $F^{-}$and $F^{+}$, define the mapping $\varphi$ on $Q$ by $\varphi(q)=\left\{y \mid y=z-\left(c^{\top} z\right) c, z \in \zeta(q)\right\}, q \in Q$. We will show that $\varphi(q) \subset A^{*}(I)$ when $q$ lies in the interior of the face $F(I)$ of $Q$. Clearly, $c^{\top} y=0$ for any $y \in \varphi(q)$. Moreover, for any $y=z-\left(c^{\top} z\right) c \in \varphi(q)$ and $i \in I(q) \cap I_{m}$ it holds that $a^{i^{\top}} y=a^{i^{\top}} z-\left(a^{i^{\top}} c\right) c^{\top} z=r^{i}(q)^{\top} z \leq 0$. Therefore, any $y \in \varphi(q)$ is an element of $A^{*}(I)$, and hence $\varphi(q) \subset A^{*}(I)$ if $q \in F(I)$. According to Theorem 3.4 there exists a connected set $C$ in $Q$ intersecting both $F^{-}$and $F^{+}$such that every point $q \in C$ is a zero point of $\varphi$, i.e., $0^{n} \in \varphi(q)$. For such a $q$ it holds that there is $z \in \zeta(q)$ satisfying $z-\left(c^{\top} z\right) c=0^{n}$. Because $p(q)^{\top} z=0$, we obtain that $\left(c^{\top} z\right)\left(p(q)^{\top} c\right)=0$. Hence, $c^{\top} z=0$, since $p(q)^{\top} c>0$. This implies that $z=0^{n}$ and therefore $0^{n} \in \zeta(q)$, inducing a constrained equilibrium. Consequently there exists a connected set of constrained equilibria linking the two faces $F^{-}$and $F^{+}$.

Finally, we show that the fundamental fixed point theorems of Browder [4] and Mas-Colell [20] can also be derived from Theorem 3.4. Browder proved the continuous function case and Mas-Colell extended the result to the upper semicontinuous correspondence case.

TheOrem 7.3. Let $P$ be an $n$-dimensional polytope and let $\varphi: P \times[0,1] \Longrightarrow P$ be any correspondence satisfying Assumption 2.1. Then the set

$$
D=\{(x, t) \in P \times[0,1] \mid x \in \varphi(x, t)\}
$$

contains a connected set $C$ such that

$$
C \cap(\mathrm{P} \times\{0\}) \neq \emptyset \text { and } C \cap(\mathrm{P} \times\{1\}) \neq \emptyset .
$$

Proof. We can rewrite the set $P \times[0,1]$ as

$$
\begin{aligned}
W=\left\{(x, t) \in \mathbb{R}^{n+1} \quad\right. & \left(a^{i \top}, 0\right)\left(x^{\top}, t\right)^{\top} \leq b_{i} \quad \forall i \in I_{m} \\
& (0, \ldots, 0,-1)\left(x^{\top}, t\right)^{\top} \leq 0 \\
& \left.(0, \ldots, 0,1)\left(x^{\top}, t\right)^{\top} \leq 1\right\} .
\end{aligned}
$$

Let $c=(0, \ldots, 0,1)^{\top} \in \mathbb{R}^{n+1}$. Obviously, $W$ is simple and no constraint is redundant. Moreover, $F^{+}=\{(x, t) \in W \mid t=1\}$ and $F^{-}=\{(x, t) \in W \mid t=0\}$.

Construct the correspondence $\psi: W \Longrightarrow \mathbb{R}^{n+1}$ as

$$
\psi(x, t)=(\varphi(x, t)-\{x\}) \times\{0\} .
$$

We will show that for any $I \in \mathcal{I}$ and any $(x, t) \in F(I)$, we have $\psi(x, t) \subset A^{*}(I)$. For $I=\emptyset, \psi(x, t) \subset A^{*}(\emptyset)$ since $c^{\top} z=0$ for any $z \in \psi(x, t)$. Now take any nonempty set $I$ from $\mathcal{I}$. We have to consider the following two cases:

(1) In case $I \subset I_{m}$, take any $(x, t)$ in the face $F(I)$ of $W$ and any $z$ in $\psi(x, t)$. We have

$$
a^{i \top} x=b_{i} \forall i \in I ; z=\left((k-x)^{\top}, 0\right)^{\top}
$$

for some $k \in P$. Take any $y \in A(I)$. That is,

$$
y=\sum_{i \in I} \lambda_{i}\left(a^{i \top}, 0\right)^{\top}+\beta c
$$


for some $\lambda_{i} \geq 0$ and $\beta \in \mathbb{R}$. Thus, we have

$$
z^{\top} y=\sum_{i \in I} \lambda_{i}(k-x)^{\top} a^{i}
$$

Since $k \in P$, we have $a^{i \top} k \leq b_{i}$ for $i \in I_{m}$. Since $a^{i \top} x=b_{i}$ for $i \in I$, we have $z^{\top} y \leq 0$. This means that $\psi(x, t)$ is a subset of $A^{*}(I)$.

(2) If $I \not \subset I_{m}$, either $m+1$ or $m+2$ is contained in $I$. For example, suppose that $m+2$ is in $I$, i.e., $t=1$. The case $m+1 \in I$ follows the same argument. Take any $(x, t)$ in the face $F(I)$ of $W$ and any $z$ in $\psi(x, t)$. We have

$$
a^{i \top} x=b_{i} \forall i \in I \backslash\{m+2\} ; t=1 ; z=\left((k-x)^{\top}, 0\right)^{\top}
$$

for some $k \in P$. Take any $y \in A(I)$. That is,

$$
y=\sum_{i \in I \backslash\{m+2\}} \lambda_{i}\left(a^{i \top}, 0\right)^{\top}+\beta c
$$

for some $\lambda_{i} \geq 0$ and $\beta \in \mathbb{R}$. Thus, we have

$$
z^{\top} y=\sum_{i \in I \backslash\{m+2\}} \lambda_{i}(k-x)^{\top} a^{i} .
$$

Since $k \in P$, we have $a^{i \top} k \leq b_{i}$ for $i \in I_{m}$. Since $a^{i \top} x=b_{i}$ for $i \in I \backslash\{m+2\}$, we have $z^{\top} y \leq 0$. This means that $\psi(x, t)$ is again a subset of $A^{*}(I)$. By Theorem 3.4 there exists a connected set $C$ in $W$ such that

$$
0^{n+1} \in \psi(x, t) \quad \forall(x, t) \in C ; F^{+} \cap C \neq \emptyset ; F^{-} \cap C \neq \emptyset .
$$

Clearly, $x \in \varphi(x, t)$ for each $(x, t) \in C$.

We remark that the above theorem can also be derived from Theorem 3.2 or Theorem 3.3.

Acknowledgment. Zaifu Yang would like to thank the CentER for Economic Research of Tilburg University for its hospitality.

\section{REFERENCES}

[1] E. L. Allgower And K. Georg, Numerical Continuation Methods: An Introduction, Springer, Berlin, 1990.

[2] J. P. Aubin, Optima and Equilibria, Springer, Berlin, 1998.

[3] L. E. J. Brouwer, Über Abbildung von Mannigfaltigkeiten, Math. Ann., 71 (1912), pp. 97-115.

[4] F. E. BRowder, On continuity of fixed points under deformations of continuous mappings, Summa Brasiliensis Mathematicae, 4 (1960), pp. 183-191.

[5] D. J. Brown, P. M. DeMarzo, and B. C. Eaves, Computing zeroes of sections of vector bundles using homotopies and relocalization, Math. Oper. Res., 21 (1996), pp. 26-43.

[6] P. M. Demarzo and B. C. Eaves, Computing equilibria of GEI by relocalization on a Grassmann manifold, J. Math. Econom., 26 (1996), pp. 479-497.

[7] B. C. Eaves, On the basic theory of complementarity, Math. Programming, 1 (1971), pp. 68-75.

[8] B. C. Eaves, Homotopies for computation of fixed points, Math. Programming, 3 (1972), pp. $1-22$.

[9] J. Freidenfelds, A set intersection theorem and applications, Math. Programming, 7 (1974), pp. 199-211.

[10] S. Fujishige And Z. YAng, A lexicographic algebraic theorem and its applications, Linear Algebra Appl., 279 (1998), pp. 75-91. 
[11] P. Hartman and G. Stampacchia, On some nonlinear elliptic differential functional equations, Acta Math., 115 (1966), pp. 271-310.

[12] P. J. J. HeRINGs, Equilibrium existence results for economies with price rigidities, Econom. Theory, 7 (1996), pp. 63-80.

[13] P. J. J. Herings, On the existence of a continuum of constrained equilibria, J. Math. Econom., 30 (1998), pp. 257-273.

[14] P. J. J. Herings and A. J. J. TAlman, Intersection theorems with a continuum of intersection points, J. Optim. Theory Appl., 96 (1998), pp. 311-335.

[15] P. J. J. Herings, A. J. J. Talman, And Z. YAng, The computation of a continuum of constrained equilibria, Math. Oper. Res., 21 (1996), pp. 675-696.

[16] S. Kakutani, A generalization of Brouwer's fixed point theorem, Duke Math. J., 8 (1941), pp. $457-459$.

[17] B. Knaster, C. Kuratowski, and C. Mazurkiewicz, Ein Beweis des Fixpunktsatzes für n-dimensionale Simplexe, Fund. Math., 14 (1929), pp. 132-137.

[18] G. van der LaAn and A. J. J. Talman, A restart algorithm for computing fixed points without an extra dimension, Math. Programming, 17 (1979), pp. 74-84.

[19] G. VAN DeR LAAn, A. J. J. TAlman, AND Z. YANG, Existence and approximation of robust solutions of variational inequality problems over polytopes, SIAM J. Control Optim., 37 (1998), pp. 333-352.

[20] A. Mas-Colell, A note on a theorem of F. Browder, Math. Programming, 6 (1974), pp. $229-233$.

[21] J. Munkres, Topology, Prentice-Hall, Englewood Cliffs, NJ, 1975.

[22] H. SCARF, The approximation of fixed points of a continuous mapping, SIAM J. Appl. Math., 15 (1967), pp. 1328-1343.

[23] S. Schalk and A. J. J. Talman, Rationing Equilibria under General Price Rigidities, mimeo, Tilburg University, The Netherlands, 2000.

[24] A. J. J. Talman and Y. Yamamoto, A simplicial algorithm for stationary point problems on polytopes, Math. Oper. Res., 14 (1989), pp. 383-399.

[25] M. J. Todd, The Computation of Fixed Points and Applications, Lecture Notes in Econom. and Math. Systems 124, Springer, Berlin, 1976.

[26] Y. Yамамото, A path-following procedure to find a proper equilibrium of finite games, Internat. J. Game Theory, 22 (1993), pp. 249-259.

[27] Z. YANG, A simplicial algorithm for computing robust stationary points of a continuous function on the unit simplex, SIAM J. Control Optim., 34 (1996), pp. 491-506.

[28] Z. YAng, Computing Equilibria and Fixed Points, Kluwer Academic Publishers, Boston, 1999. 